\title{
MAGNETO-ACOUSTIC WAVES IN A GRAVITATIONALLY STRATIFIED MAGNETIZED PLASMA: EIGEN-SOLUTIONS AND THEIR APPLICATIONS TO THE SOLAR ATMOSPHERE
}

\author{
J. F. MATHER ${ }^{1}$ and R. ERdÉLYI ${ }^{1,2}$ \\ ${ }^{1}$ Solar Physics and Space Plasma Research Centre (SP2RC), School of Mathematics and Statistics, University of Sheffield, Hicks Building, \\ Hounsfield Road, Sheffield, S3 7RH, UK \\ ${ }^{2}$ Debrecen Heliophysical Observatory (DHO), Konkoly Observatory, Research Centre for Astronomy and Earth Sciences, Hungarian Academy of Sciences, H-4010 \\ Debrecen, P.O. Box 30, Hungary; robertus@ sheffield.ac.uk \\ Received 2016 January 25; accepted 2016 February 24; published 2016 May 12
}

\begin{abstract}
Magneto-acoustic gravity (MAG) waves have been studied intensively in the context of astrophysical plasmas. There are three popular choices of analytic modeling using a Cartesian coordinate system: a magnetic field parallel, perpendicular, or at an angle to the gravitational field. Here, we study a gravitationally stratified plasma embedded in a parallel, so called vertical, magnetic field. We find a governing equation for the auxiliary quantity $\Theta=p_{1} / \rho_{0}$, and find solutions in terms of hypergeometric functions. With the convenient relationship between $\Theta$ and the vertical velocity component, $v_{z}$, we derive the solution for $v_{z}$. We show that the four linearly independent functions for $v_{z}$ can also be cast as single hypergeometric functions, rather than the Frobenius series derived by Leroy \& Schwartz. We are then able to analyze a case of approximation for a one-layer solution, taking the small wavelength limit. Motivated by solar atmospheric applications, we finally commence study of the eigenmodes of perturbations for a two-layer model using our solutions, solving the dispersion relation numerically. We show that, for a transition between a photospheric and chromospheric plasma embedded in a vertical magnetic field, modes exist that are between the observationally widely investigated three and five minute oscillation periods, interpreted as solar global oscillations in the lower solar atmosphere. It is also shown that, when the density contrast between the layers is large (e.g., applied to photosphere/chromosphere-corona), the global eigenmodes are practically a superposition of the same as in each of the separate one-layer systems.
\end{abstract}

Key words: magnetohydrodynamics (MHD) - Sun: chromosphere - Sun: corona - Sun: magnetic fields - waves

\section{INTRODUCTION}

Waves and oscillations have been routinely observed in the Sun, most notably at the well known global acoustic five minute oscillations. Cowling (1941) considered the Sun as a spherically symmetric body and, accounting for the stratification due to gravity, found that two types of global oscillations exist: $p$-modes and $g$-modes. These oscillations can be mathematically modeled using spherical harmonics, $Y_{l}^{m}$, where $l$ is the degree of the oscillation and $m$ is the azimuthal order. $p$-modes account for the widely observed five minute periods and are pressure-driven waves modified, slightly, by gravity, whereas $g$-modes are oscillations driven by buoyant motions. The internal gravity modes have never been categorically observed, in part due to their long periods, their low signal-to-noise, and also their evanescent behavior in, and above, the convection zone. At the solar interior-atmosphere interface, the $f$-modes exist as surface modes, having the largest amplitude at the solar surface. They are gravitational in nature and exist in compressible, as well as incompressible, plasmas.

When considering a plasma with an embedded magnetic field within a gravitational field, the geometry of the background equilibrium can significantly increase the complexity of the mathematics that model MHD waves. In a Cartesian coordinate system (taking the $z$-direction parallel to the gravitational field), the direction of the magnetic field in comparison to the gravitational field has important consequences. When the magnetic field is perpendicular to the gravitational field the overall governing equations for perturbations, in the direction of inhomogeneity, are second order differential equations. For examples of work on horizontal fields see e.g., Miles \& Roberts (1992), Pintér (1999), and
Goedbloed \& Poedts (2004). However, if the magnetic field is taken to be parallel to the gravitational field, two governing equations are obtained. One is the governing equation associated with the motion of the Alfvén waves in a stratified plasma. The second is a fourth-order equation describing the wave motions of both "slow" and "fast" waves. When the background magnetic field has both a parallel and perpendicular component, compared to the gravitational field, the governing equation for perturbations has a highest derivative of a sixth order in the direction of inhomogeneity. One finds that the Alfvén wave can no longer be decoupled from the slow and fast waves.

A number of authors have studied the oscillations in a solar model with stratification and parallel magnetic field or "vertical field" as it is more commonly called. Analytic solutions in isothermal plasmas have been readily derived. Ferraro \& Plumpton (1958) showed that the magneto-acoustic gravity (MAG) waves could be described by a fourth order governing equation for the horizontal component of the velocity perturbation $\left(v_{x}\right)$. They were able to find series solutions to this equation using the Fröbenius method. Zhugzhda (1979), again, showed it was possible to find series solution for $v_{x}$ using the Fröbenius method. The work was, however, extended to show these solutions could be written as the more general Meijer-G functions, as well as hypergeometric functions (for small argument). Using these solutions, it was found that the slow wave degenerates to a vertically propagating sound wave along the magnetic field lines and that the fast wave is evanescent in the low-beta limit. Leroy \& Schwartz (1982) derived a series solution for $v_{x}$, but also found a series solution for the vertical (parallel to magnetic field) velocity component 
$\left(v_{z}\right)$. They implemented and derived boundary conditions for a plasma-plasma interface model. Cally (2001) re-asserted the hypergeomtric solutions obtained by Zhugzhda (1979) and also extended the work of Zhugzhda \& Dzhalilov (1982) on the transmission and reflection coefficients of MAG waves propagating into the $\beta \approx 1$ layer. However, none of these authors were able to find $v_{z}$ in terms of special functions. The current study finds it is possible to write the linearly independent solutions describing $v_{z}$ as single hypergeometric functions, while also finding a hypergeometric function description for $\Theta$ analytically. This is an important finding, since actually $v_{z}$ (well, strictly speaking the line of sight component of velocity) is observed.

In terms of modeling global oscillations of the solar atmosphere, given a vertical equilibrium magnetic field, there has been little fully analytical success to find eigen-frequencies, due to the complexity of the problem. One notable exception is the work by Hasan \& Christensen-Dalsgaard (1992). The authors studied a one-layer bounded atmosphere employed in a high-beta limit of the dispersion relation, using asymptotic expansions for the Meijer-G functions and a correction method to find the eigen-frequencies. It was found that with the introduction of a magnetic field, even a small one, nullified the $g$-modes. In a follow up work Banerjee et al. (1995) studied a one-layer bounded atmosphere for the high-beta case but for zero derivative boundary conditions in the top layer. This complexity of analytical study has driven many to study the problem numerically or semi-analytically (for recent studies see e.g., Fedun et al. 2009, 2011).

Scheuer \& Thomas (1981) modeled oscillations in sunspots, numerically, using two-layer and three-layer cylindrical models. They found that resonant "fast" modes existed for periods typical of sunspot umbral oscillations. In a companion paper to Leroy \& Schwartz (1982), MAG oscillations were studied numerically, modeling propagating perturbations from the photosphere to corona. They found that it was unlikely these modes would penetrate deep into the corona with any considerable energy (Schwartz \& Leroy 1982). Abdelatif (1990) investigated the avoided crossings of the coupled "slow" and "fast" solutions for a line-tied atmosphere. It was found that away from the avoided crossings the solutions could be described by the uncoupled "slow" and "fast" wave solutions. It was also found that enforcing the derivative of the vertical component of the velocity to be zero at the boundaries changed the nature of these avoided crossings. Yelles Chaouche \& Abdelatif (2005) investigated a two-layer plasma, unbounded at the top and stratified by gravity, using the solutions obtained by Leroy \& Schwartz (1982). It was found that this particular model introduced a positive imaginary component to the horizontal wavenumber that damped waves propagating perpendicular to the magnetic field. The second part of this article studies two representative two plasma layer models: a photosphere to chromosphere transition and a lower solar atmosphere to corona transition.

The outline of this paper is as follows: in Section 2 the background equilibrium for the problem is defined. In Section 3 the linearized equations are presented. The governing equation for the variable $\Theta=p_{1} / \rho_{0}$ is then derived and solutions for this variable are given. Here, $\Theta$ is the ratio of the pressure perturbation to the background density. Solutions for the horizontal $\left(v_{x}\right)$ and vertical $\left(v_{z}\right)$ velocity perturbations are derived. In Section 4 the single-layer model dispersion relation is attained. Next, the small wavelength limit of this dispersion relation is taken. In Section 5 the two-layer model is introduced along with its own "background." Two different solar representations are then studied to find dispersion relations and the wave energy in these eigenmodes is found. Finally, the main conclusions of the work are summarized in Section 6.

\section{BACKGROUND AND LINEARIZED EQUATIONS}

Consider an ideal plasma, one that is perfectly conductive and acts as an ideal gas. The curved nature of the Sun is approximated in terms of Cartesian coordinates. The plasma is stratified by a gravitational field pointing in the negative $z$ direction and is embedded in a parallel uniform magnetic field. The plasma, then, conforms to the ideal gas law and the ideal MHD equations. There are no sources of heating and the plasma is adiabatic and assumed to be isothermal (no background temperature inhomogeneity). There are no background flows either (i.e., $v_{0}=0$ ). The variations of background quantities compared to the time-scale of characteristic wave motions is negligible such that $\frac{\partial}{\partial t} \approx 0$ of any background quantity. The medium is infinite and homogeneous in the horizontal (i.e., parallel to the solar surface) $x$ - and $y$-directions. The magnetic field takes the form of $\boldsymbol{B}_{0}=\left(0,0, B_{0}\right)$, where $B_{0}$ is a constant. The gravitational force is of the form $g=(0,0$, $-g$ ). The background quantities $p_{0}(z)$ and $\rho_{0}(z)$ are functions of $z$ only and in an isothermal atmosphere have the form,

$$
p_{0}=p_{0}(0) e^{-z / H}, \quad \rho_{0}=\rho_{0}(0) e^{-z / H}, \quad H=\frac{v_{s}^{2}}{\gamma g},
$$

where $H$ is the pressure scale height, $\gamma$ is the ratio of specific heats, and $v_{s}$ is the sound speed. Two characteristic speeds that often appear for wave motions are:

$$
v_{s}^{2}=\frac{\gamma p_{0}}{\rho_{0}}=\mathrm{const}, \quad v_{A}^{2}=\frac{B_{0}^{2}}{\mu_{0} \rho_{0}}=v_{A 0}^{2} e^{z / H} .
$$

Here, $\mu_{0}$ is the magnetic permeability of free space and $v_{A}$ is the Alfvén speed. The background is depicted in Figure 1. The linearized ideal MHD equations are assumed to hold if one takes small perturbations around this background plasma. These governing equations are presented below:

$$
\begin{gathered}
\frac{\partial \rho_{1}}{\partial t}+\left(\boldsymbol{v}_{1} \cdot \nabla\right) \rho_{0}+\rho_{0}\left(\nabla \cdot \boldsymbol{v}_{1}\right)=0, \\
\frac{\partial p_{1}}{\partial t}+\left(\boldsymbol{v}_{1} \cdot \nabla\right) p_{0}+\gamma p_{0}\left(\nabla \cdot \boldsymbol{v}_{1}\right)=0, \\
\rho_{0} \frac{\partial \boldsymbol{v}_{1}}{\partial t}=-\nabla p_{1}+\left(\nabla \times \boldsymbol{B}_{1}\right) \times \frac{\boldsymbol{B}_{0}}{\mu}-\rho_{1} g \hat{z}, \\
\frac{\partial \boldsymbol{B}_{1}}{\partial t}=\nabla \times\left(\boldsymbol{v}_{1} \times \boldsymbol{B}_{0}\right), \quad \nabla \cdot \boldsymbol{B}_{1}=0 .
\end{gathered}
$$

\section{PERTURBATION EQUATIONS AND THEIR SOLUTIONS}

\subsection{Previous Work}

Because the background is homogeneous in the $x$ - and $y$-directions, the perturbed variables can therefore be Fourier decomposed and written in the form $f(x, y, z, t)=$ $f_{1}(z) e^{i\left(k_{x} x+k_{y} y-\omega t\right)}$. Since the only preferred direction in the system is the $z$-direction, the coordinate system can be rotated 
such that $k_{y}=0$ (without loss of generality), then $f(x, z, t)=f_{1}(z) e^{i\left(k_{x} x-\omega t\right)}$. In what follows, we drop the subscript " $x$ " and use $k$ instead of $k_{x}$. The linearized MHD equations take the form of three equations for the variables $v_{x}$, $v_{y}$, and $v_{z}$, respectively:

$$
\begin{gathered}
v_{A}^{2}(z) \frac{d^{2} v_{x}}{d z^{2}}+\left(\omega^{2}-k_{x}^{2}\left(v_{s}^{2}+v_{A}^{2}(z)\right)\right) v_{x} \\
=i k_{x} g v_{z}-i k_{x} v_{s}^{2} \frac{d v_{z}}{d z}, \\
v_{A}^{2}(z) \frac{d^{2} v_{y}}{d z^{2}}+\omega^{2} v_{y}=0, \\
v_{s}^{2} \frac{d^{2} v_{z}}{d z^{2}}-\gamma g \frac{d v_{z}}{d z}+\omega^{2} v_{z}=i(\gamma-1) g k_{x} v_{x}-i v_{s}^{2} k_{x} \frac{d v_{x}}{d z} .
\end{gathered}
$$

Taking the limit $k_{x}=0$, Equations (7) and (9) form the well known equations, see, e.g., Hasan \& Christensen-Dalsgaard (1992),

$$
\begin{gathered}
v_{A}^{2}(z) \frac{d^{2} v_{x}}{d z^{2}}+\omega^{2} v_{x}=0, \\
v_{s}^{2} \frac{d^{2} v_{z}}{d z^{2}}-\gamma g \frac{d v_{z}}{d z}+\omega^{2} v_{z}=0 .
\end{gathered}
$$

It is easy to see that Equation (10) governs a "magnetic" wave and Equation (11) governs an acoustic wave. In addition, the velocity components, $v_{x}$ and $v_{z}$ have been decoupled from one another. Equations (10) and (11) permit the following solutions for $v_{x}$ and $v_{z}$, respectively:

$$
\begin{gathered}
v_{x}=C_{1} J_{0}(2 \xi)+C_{2} Y_{0}(2 \xi), \\
v_{z}=C_{3} \xi^{-1+2 i \sqrt{\Omega^{2} / \gamma-1 / 4}}+C_{4} \xi^{-1-2 i \sqrt{\Omega^{2} / \gamma-1 / 4}},
\end{gathered}
$$

where $J_{O}$ and $Y_{0}$ are Bessel's functions of the first and second kind of zeroth order, respectively. Here, dimensionless variables have been introduced and are given below (note, these will be used throughout this article):

$$
\xi=\frac{\omega H}{v_{A}}, \quad \Omega=\frac{\omega H \sqrt{\gamma}}{v_{s}}, \quad K=k_{x} H .
$$

Considering the more general case when $k_{x} \neq 0$, Equations (7) and (9) can be used to derive a dimensionless differential equation for $v_{x}$, see e.g., Zhugzhda (1979):

$$
\begin{gathered}
\xi^{4} \frac{d^{4} v_{x}}{d \xi^{4}}+4 \xi^{3} \frac{d^{3} v_{x}}{d \xi^{3}}+\xi^{2}\left[\xi^{2}+1+4\left(\frac{\Omega^{2}}{\gamma}-K^{2}\right)\right] \frac{d^{2} v_{x}}{d \xi^{2}} \\
+\xi\left[3 \xi^{2}-1+4\left(\frac{\Omega^{2}}{\gamma}+K^{2}\right)\right] \frac{d v_{z}}{d \xi} \\
+16\left[\left(\frac{\Omega^{2}}{\gamma}+K^{2}\left(\frac{\gamma-1}{\gamma \Omega^{2}}-1\right)\right) \xi^{2}-\frac{4 K^{2} \Omega^{2}}{\gamma}\right] v_{x}
\end{gathered}
$$$$
=0 \text {. }
$$

Equation (14) can be solved with hypergeometric solutions, see, e.g., Equation (8) in Cally (2001). The solution to
Equation (14) is given below as

$$
\begin{aligned}
& v_{x} \\
& =A_{1} \xi^{2 K}{ }_{2} F_{3}\left[\begin{array}{l|l}
\frac{1}{2}-i q_{z}+K, \frac{1}{2}+i q_{z}+K ; & -\xi^{2} \\
1+2 K, \frac{1}{2}-i q_{0}+K, \frac{1}{2}+i q_{0}+K &
\end{array}\right] \\
& +A_{2} \xi^{-2 K_{2} F_{3}}\left[\begin{array}{l|l}
\frac{1}{2}+i q_{z}-K, \frac{1}{2}-i q_{z}-K ; \\
1-2 K, \frac{1}{2}-i q_{0}-K, \frac{1}{2}+i q_{0}-K
\end{array} \mid-\xi^{2}\right. \\
& +A_{3} \xi^{1+i 2 q_{0}}{ }_{2} F_{3} \\
& \times\left[\begin{array}{l|l}
1+i q_{0}-i q_{z}, 1+i q_{0}+i q_{z} ; \\
1+2 i q_{0}, \frac{3}{2}+i q_{0}+K, \frac{3}{2}+i q_{0}-K
\end{array}\right] \\
& +A_{4} \xi^{1-i 2 q_{0}}{ }_{2} F_{3} \\
& \times\left[\begin{array}{l}
1-i q_{z}-i q_{0}, 1+i q_{z}-i q_{0} ; \\
1-2 i q_{0}, \frac{3}{2}-i q_{0}+K, \frac{3}{2}-i q_{0}-K
\end{array}\right]
\end{aligned}
$$

The quantities $q_{z}$ and $q_{0}$ are defined as

$$
\begin{aligned}
& q_{z}=\sqrt{\frac{\Omega^{2}}{\gamma}-\frac{\left(\Omega^{2}-\hat{N}^{2}\right) K^{2}}{\Omega^{2}}-\frac{1}{4}}, \\
& q_{0}=\sqrt{\frac{\Omega^{2}}{\gamma}-\frac{1}{4}, \quad \hat{N}^{2}=\frac{\gamma-1}{\gamma} .}
\end{aligned}
$$

Here $\hat{N}$ is the dimensionless Brunt-Väisälä frequency in an isothermal plasma (the frequency at which a fluid parcel would oscillate around its equilibrium position). The dimensional from is shown in Appendix.

\subsection{Alternative Solutions}

As has already been stated, solutions were found earlier for $v_{x}$. Next, the vertical velocity component can be written in terms of derivatives of $v_{x}$ up to the third order in $\xi$. In this article we aim to show that, by defining a new and suitably chosen auxiliary variable, a single hypergeometric function solution can be derived for $v_{z}$. Let us introduce $\Theta=p_{1} / \rho_{0}$, as the equations connecting $v_{x}$ and $\Theta$, as well as $v_{z}$ and $\Theta$, are substantially simpler. The fourth-order differentials are found for $\Theta$ (a detailed derivation is left for the Appendix.

$$
\begin{aligned}
& \xi^{4} \frac{d^{4} \Theta}{d \xi^{4}}+8 \xi^{3} \frac{d^{3} \Theta}{d \xi^{3}}+\xi^{2}\left[4 \xi^{2}+13+4\left(\frac{\Omega^{2}}{\gamma}-K^{2}\right)\right] \frac{d^{2} \Theta}{d \xi^{2}} \\
& +\xi\left[12 \xi^{2}+3+4 \frac{\Omega^{2}}{\gamma}-12 K^{2}\right] \frac{d \Theta}{d \xi} \\
& +16\left[\left(\frac{\Omega^{2}}{\gamma}+K^{2}\left(\frac{\gamma-1}{\gamma \Omega^{2}}-1\right)\right) \xi^{2}-\frac{K^{2} \Omega^{2}}{\gamma}\right] \Theta=0 .
\end{aligned}
$$

Using the Fröbenius method, solutions to Equation (16) can be written as an infinite series. These series solutions can then 
be written as hypergeometric functions.

$$
\begin{aligned}
& \Theta=C_{1} \xi^{2 K_{2} F_{3}}\left[\begin{array}{c|c}
a_{11}, a_{12} ; \\
b_{11}, b_{12}, b_{13}
\end{array} \mid-\xi^{2}\right] \\
& +C_{2} \xi^{-2 K_{2} F_{3}}\left[\begin{array}{c}
a_{21}, a_{22} ; \\
b_{21}, b_{22}, b_{23}
\end{array} \mid-\xi^{2}\right] \\
& +C_{3} \xi^{-1+i 2 q_{0}}{ }_{2} F_{3}\left[\begin{array}{c}
a_{31}, a_{32} ; \\
b_{31}, b_{32}, b_{33}
\end{array} \mid-\xi^{2}\right] \\
& +C_{4} \xi^{-1-i 2 q_{0}}{ }_{2} F_{3}\left[\begin{array}{c}
a_{41}, a_{42} ; \\
b_{41}, b_{42}, b_{43}
\end{array} \mid-\xi^{2}\right] \text {. }
\end{aligned}
$$

Note that an arbitrary hypergeometric function of the variable $-\xi^{2}$ can be written as

$$
{ }_{p} F_{q}\left[\begin{array}{c}
a_{1}, \ldots, a_{p} ; \\
b_{1}, \ldots, b_{q}
\end{array} \mid-\xi^{2}\right]=\sum_{n=0}^{\infty} \frac{\left(a_{1}\right)_{n} \ldots\left(a_{p}\right)_{n}\left(-\xi^{2}\right)^{n}}{\left(b_{1}\right)_{n} \ldots\left(b_{q}\right)_{n} n !}
$$

where the Pochammer symbol, $(a)_{n}$, is defined

$$
(a)_{n}=(a)(a+1) \ldots(a+(n-1)), \quad(a)_{0}=1, \quad \forall a \in \mathbb{C},
$$

with $\mathbb{C}$ denoting the set of complex numbers. Solutions for both $v_{x}$ and $v_{z}$ can then be found in a straightforward way using the relationships between $\Theta, v_{x}$, and $v_{z}$, respectively. Detailed derivations and definitions of $a_{i j}, b_{i j}(i, j=1, . .4)$ can be found in Appendix B. The solutions for $v_{x}$ and $v_{z}$ are presented below:

$$
\begin{aligned}
& v_{x}=C_{1} \xi^{2 K_{S}(1)}{ }_{2} F_{3}\left[\begin{array}{c}
a_{11}, a_{12} ; \\
b_{11}, b_{12}-1, b_{13}-1
\end{array} \mid-\xi^{2}\right] \\
& +C_{2} \xi^{-2 K} S^{(2)}{ }_{2} F_{3}\left[\begin{array}{c}
a_{21}, a_{22} ; \\
b_{21}, b_{22}-1, b_{23}-1
\end{array} \mid-\xi^{2}\right] \\
& -C_{3} \xi^{1+2 i q_{0}} S^{(3)}{ }_{2} F_{3}\left[\begin{array}{c}
a_{31}+1, a_{32}+1 ; \\
b_{31}, b_{32}+1, b_{33}+1
\end{array} \mid-\xi^{2}\right] \\
& -C_{4} \xi^{1-2 i q_{0}} S^{(4)}{ }_{2} F_{3}\left[\begin{array}{c}
a_{41}+1, a_{42}+1 ; \\
b_{41}, b_{42}+1, b_{43}+1
\end{array} \mid-\xi^{2}\right] \text {, }
\end{aligned}
$$

$$
\begin{aligned}
& v_{z}=i K
\end{aligned}
$$

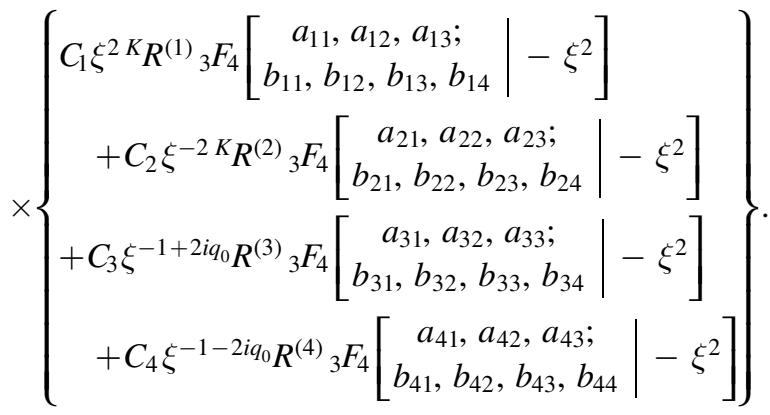

Note that $S^{(i)}, R^{(i)}, a_{i j}$, and $b_{i j}$ are given in Appendix B. Equation (18) is none other than the solution given by Equation (15). The solution for $v_{z}$, given by Equation (19), does not differ in essence from the solution given in Leroy \& Schwartz (1982). The difference here is that we have shown that the solution for $v_{z}$ can be written in the form of hypergeometric functions, rather than just a Fröbenius series. This simplifies analysis and computation. Generalized hypergeometric functions have been well studied and various asymptotic expansions have been derived for large and small arguments, see e.g., Luke (1975). With these asymptotic relations we can see the physics of the problem more readily than could be seen with the Fröbenius series.

\section{SINGLE LAYER}

Before looking at the far more complicated problem of a multi-layer atmospheric plasma model, it is expedient to study the normal modes of oscillation of a single-layer bounded plasma. The single atmospheric plasma layer model has been addressed in several different ways by e.g., Hasan \& Christensen-Dalsgaard (1992) or Abdelatif (1990). The boundary conditions that are imposed are line-tying at the boundaries (perfect reflection), i.e., $v_{x}=v_{z}=0$ at $z=0, D_{0}$. The four boundary conditions permit four linearly independent equations that may be written in a matrix form to determine the coefficients $C_{1}, C_{2}, C_{3}$, and $C_{4}$ :

$$
\left(\begin{array}{cccc}
v_{x(0)}^{(1)} & v_{x(0)}^{(2)} & v_{x(0)}^{(3)} & v_{x(0)}^{(4)} \\
v_{x\left(D_{0}\right)}^{(1)} & v_{x\left(D_{0}\right)}^{(2)} & v_{x\left(D_{0}\right)}^{(3)} & v_{x\left(D_{0}\right)}^{(4)} \\
v_{z(0)}^{(1)} & v_{z(0)}^{(2)} & v_{z(0)}^{(3)} & v_{z(0)}^{(4)} \\
v_{z\left(D_{0}\right)}^{(1)} & v_{z\left(D_{0}\right)}^{(2)} & v_{z\left(D_{0}\right)}^{(3)} & v_{z\left(D_{0}\right)}^{(4)}
\end{array}\right)\left(\begin{array}{l}
C_{1} \\
C_{2} \\
C_{3} \\
C_{4}
\end{array}\right)=0 .
$$

Note that here $v_{x(a)}^{(j)}$ and $v_{z(a)}^{(j)}\left(a=0, D_{0}\right.$, and $\left.j=1,2,3,4\right)$ refer to the functions multiplying the constants of integration, $C_{j}$. Here, $D_{0}$ is the height of the upper boundary of the atmosphere. To obtain non-trivial solutions, the determinant of the matrix on the left is taken to obtain the full dispersion relation for standing waves in a bounded plasma with a vertical field that is stratified by a vertical gravitational force.

\subsection{Small Wavelength Limit $\left(k_{x} \rightarrow \infty\right)$}

Taking the limit of the determinant of Equation (20) as $k_{x} \rightarrow \infty$, one arrives at,

$$
\left(v_{z(0)}^{3} v_{4\left(D_{0}\right)}^{4}-v_{z\left(D_{0}\right)}^{3} v_{z(0)}^{4}\right)=0 .
$$

Here, the functions $v_{z}^{(3)}$ and $v_{z}^{(4)}$ are given by

$$
\begin{aligned}
& v_{\left(0, D_{0}\right)}^{(3)}=\xi_{\left(0, D_{0}\right)}^{-1+2 i q_{0}} R^{(3)}{ }_{1} F_{2}\left[\begin{array}{c}
a_{33} ; \\
b_{31}, b_{34}
\end{array} \mid-\frac{\left(\Omega^{2}-\hat{N}^{2}\right) \xi_{(0, L)}^{2}}{\Omega^{2}}\right], \\
& v_{\left(0, D_{0}\right)}^{(4)}=\xi_{\left(0, D_{0}\right)}^{-1-2 i q_{0}} R^{(4)}{ }_{1} F_{2}\left[\begin{array}{c}
a_{43} ; \\
b_{41}, b_{44}
\end{array} \mid-\frac{\left(\Omega^{2}-\hat{N}^{2}\right) \xi_{\left(0, D_{0}\right)}^{2}}{\Omega^{2}}\right] .
\end{aligned}
$$

It is well known that taking this limit in Equations (7) and (8) removes the fast mode (see, e.g., Moreno-Insertis \& Spruit 1989; Roberts 2006) and this is exactly what is seen here. If we take the limit of small plasma-beta, that is $\beta \ll 1$, then the parameter $\xi^{2} \ll 1$. Therefore, the hypergeometric function ${ }_{1} F_{2}\left[;-\frac{\left(\Omega^{2}-\hat{N}^{2}\right) \xi_{\left(0, D_{0}\right)}^{2}}{\Omega^{2}}\right] \approx 1$, and Equation (21) becomes

$$
\sin \left(q_{0} D_{0} / H\right)=0, \quad \frac{q_{0} D_{0}}{H}=m \pi, \quad m \in \mathbb{N} .
$$


Transforming back to dimensional quantities,

$$
\frac{\omega^{2}}{v_{s}^{2}}=\frac{m^{2} \pi^{2}}{D_{0}^{2}}+\frac{1}{4 H^{2}} .
$$

Dispersion relation (24) shows that the slow mode has degenerated to a vertically propagating sound wave along the field lines. Equation (24) also reveals that the atmosphere has a cut-off frequency of $\omega_{a c}^{2}=v_{s}^{2} / 4 H^{2}$, otherwise called the acoustic cut-off frequency. If the frequencies of the perturbations fall below this, the waves are no longer standing modes.

The limit of large plasma-beta $(\beta \gg 1)$ is suitable as an approximation of the lower solar atmosphere. Therefore, taking the limit of large plasma-beta in Equation (21) (assuming $D_{0}=-D^{2}$, where $D \in \mathbb{R}$ ), the asymptotic expansion of the hypergeometric functions is taken as $\xi^{2} \rightarrow \infty$ (Luke 1975). Therefore, to the lowest order in $\xi^{-1}$, Equation (22) can be written,

$$
\begin{aligned}
& v_{\left(0, D_{0}\right)}^{(3)}=\frac{\Gamma\left(1+2 i q_{0}\right)}{2 \sqrt{\pi}} \xi_{\left(0, D_{0}\right)}^{(-1 / 2)}\left[\begin{array}{c}
e^{-i \frac{\pi}{4}} e^{q_{0}} e^{-2 i \sqrt{\left(\Omega^{2}-\hat{N}^{2}\right) / \Omega^{2}} \xi_{\left(0, D_{0}\right)}} \\
+e^{i \frac{\pi}{4}} e^{-q_{0}} e^{2 i \sqrt{\left(\Omega^{2}-\hat{N}^{2}\right) / \Omega^{2}} \xi_{\left(0, D_{0}\right)}}
\end{array}\right], \\
& v_{\left(0, D_{0}\right)}^{(4)}=\frac{\Gamma\left(1-2 i q_{0}\right)}{2 \sqrt{\pi}} \xi_{\left(0, D_{0}\right)}^{(-1 / 2)}\left[\begin{array}{c}
e^{-i \frac{\pi}{4}} e^{-q_{0}} e^{-2 i \sqrt{\left(\Omega^{2}-\hat{N}^{2}\right) / \Omega^{2}} \xi_{\left(0, D_{0}\right)}} \\
+e^{i \frac{\pi}{4}} e^{q_{0}} e^{2 i \sqrt{\left(\Omega^{2}-\hat{N}^{2}\right) / \Omega^{2}} \xi_{\left(0, D_{0}\right)}}
\end{array}\right] .
\end{aligned}
$$

Here, $\Gamma(x)$ is the gamma-function. Substituting these expressions into Equation (21) the dispersion relation reduces to

$$
\sin \left(2 \hat{\xi} \sqrt{\left(\Omega^{2}-\hat{N}^{2}\right) / \Omega^{2}}\right)=0, \quad \hat{\xi}=\xi_{D_{0}}-\xi_{0} .
$$

Clearly, for this to be zero, we require,

$$
\begin{aligned}
& 2 \hat{\xi} \sqrt{\left(\Omega^{2}-\hat{N}^{2}\right) / \Omega^{2}}=m \pi, \quad m \in \mathbb{N}, \\
& \Omega^{2}=\hat{N}^{2}+\frac{m^{2} \pi^{2}}{2 s^{2} \beta}, \quad s=1-e^{-D^{2} / 2} .
\end{aligned}
$$

It is interesting to note that Equation (26) agrees with the counterpart derived, in a completely different way, by Hasan \& Christensen-Dalsgaard (1992). These authors took the limit $k_{x} \rightarrow \infty$ of the wave equations and the high-beta limit directly implemented in the governing equation. From there, they applied boundary conditions on the solutions and again found asymptotic expansions for $\beta \gg 1$. The solution in Equation (26) has two different interpretations: the first being for low harmonics, where the solution is the $g$-modes modified by the magnetic field. However, for larger harmonics, the contribution from the Brunt-Väisälä frequency becomes negligible, thus the modes are far more magnetic in nature. Also noting the completely hydrodynamic limit, $\beta \rightarrow \infty$, we see that all modes tend to the Brunt-Väisälä frequency, i.e., the limit of $g$-modes as $k_{x} \rightarrow \infty$.

\subsection{Solar Atmospheric Models}

The aim is to investigate models reminiscent of the global solar atmosphere. The first model we opt for is one analogous to the solar corona, and the second approximates the solar photosphere and chromosphere as one isothermal layer. The first model has a main characteristic corresponding to a lowbeta plasma. The representative parameters for the first model

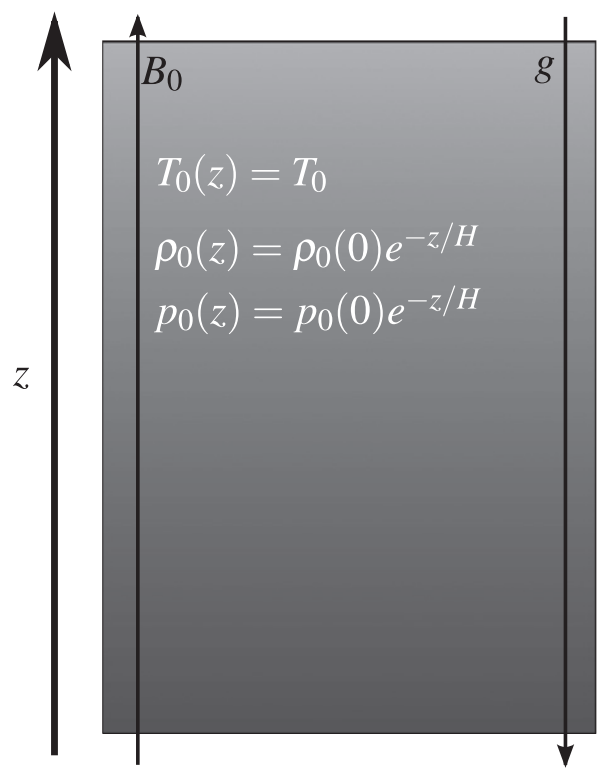

Figure 1. The equilibrium background plasma analyzed in this section. The grayscale depicts the density and pressure decrease with increasing height.

are:

$$
z=\left[0, D_{2}\right], \quad \frac{D_{2}}{H}=3,\left.\quad \beta\right|_{z=0}=0.1 .
$$

A dispersion diagram is plotted for this model in Figure 2(a).

The second solar atmospheric model is one transitioning from a high-beta plasma to a low-beta plasma as height increases, analogous to a basic model of the real solar atmosphere. Typical parameters for this particular model are:

$$
z=\left[-D_{1}, 0\right], \quad \frac{D_{1}}{H}=7,\left.\quad \beta\right|_{z=0}=0.1 .
$$

A dispersion diagram for this set up is shown in Figure 2(b), relating frequency and wavenumber.

Both these models have been included as we wish to investigate a two-layer model in Section 5. The two layers in this model will be connected by a discontinuity in density of a factor of 400, representative of the narrow Transition Region. Here we briefly elaborate on how this discontinuity affects the dispersion of MHD perturbations in the lower layer (Figure 2(b)) along with periodic perturbations in the upper layer (Figure 2(a)). Investigating the single-layer models allows for a direct comparison to the two-layer model.

With the parameters of the two separate models above, the determinant of Equation (20) is solved numerically for a series of values of $k_{x}$. Introduced below are a new dimensionless frequency $(\hat{\omega})$ and dimensionless horizontal wavenumber $\left(\hat{k}_{x}\right)$ that relate to the two-layer model,

$$
\hat{k_{x}}=k_{x}\left(D_{1}+D_{2}\right), \quad \hat{\omega}=\frac{\omega\left(D_{1}+D_{2}\right)}{v_{s_{U}}} .
$$

In Figure 2, solutions of the determinant of Equation (20) are plotted as $\hat{\omega}-\hat{k}_{x}$ dispersion diagrams. Solutions are shown near to perturbations with 3-5 minute periods. First, one can conclude that global standing wave solutions exist at periods within this range for both models. The photospheric/chromospheric sound speed is taken to be $7.9 \mathrm{~km} \mathrm{~s}^{-1}$ for Figure 2(a) (Scheuer \& Thomas 1981) and its coronal counterpart $158 \mathrm{~km} \mathrm{~s}^{-1}$ is used for Figure 2(b). Each line in the respective diagrams corresponds to a different 


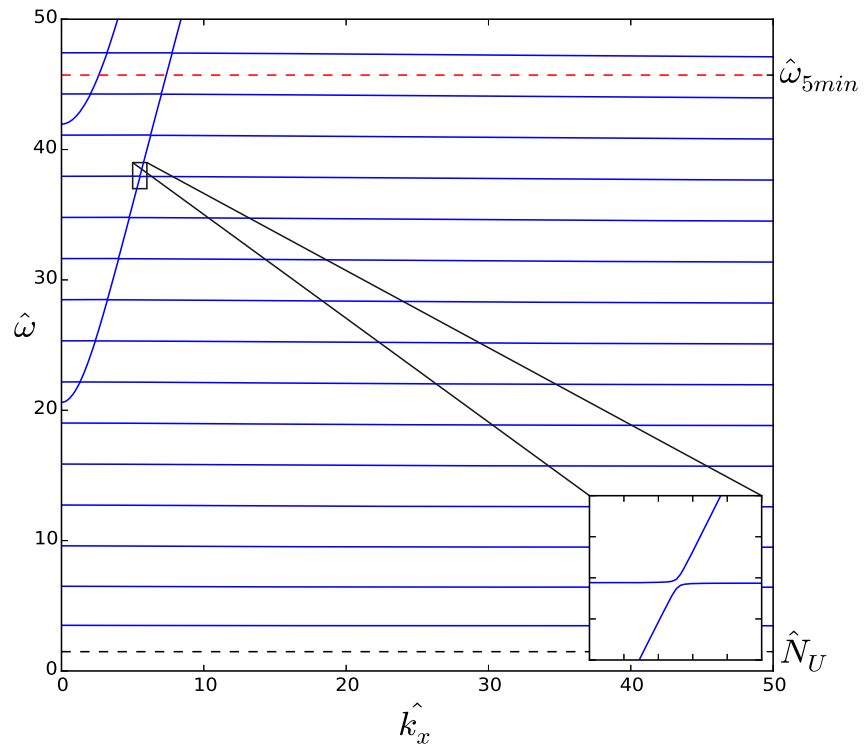

(a)

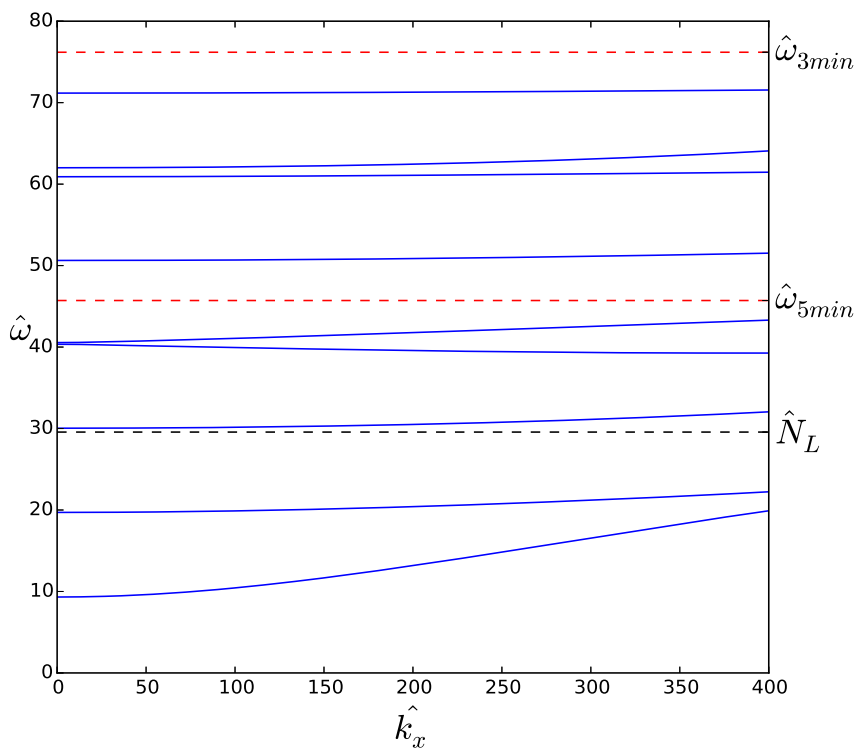

(b)

Figure 2. The dispersion diagram from the determinant of Equation (20) for (a) on the left for a low-beta plasma: $\left.\beta\right|_{z=0}=0.1$ at $z=0$ and $D_{2} / H=3$. On the right (b) the dispersion diagram for the high- to low-beta plasma described in Section 4.2: $\left.\beta\right|_{z=0}=0.1$ and $D_{1} / H=7$. The dotted lines correspond to five minute $\left(\hat{\omega}_{5}\right.$ min $)$, three minute $\left(\hat{\omega}_{3 \mathrm{~min}}\right)$ frequencies, and the Brunt-Väisäla frequencies $\left(\hat{N}_{U}\right.$ and $\hat{N}_{L}$ in the top and bottom layer, respectively).

mode number. However, at the avoided crossings (an example is zoomed in on, see Figure 2(a)), two solutions' (modes') paths meet and then separate with one solution following the previous trajectory of the other and vice-versa. Each solution then takes on the character and mode number of the other.

One can conclude from Figure 2(a) that modes exhibiting frequencies within the bands of three and five minute periods can exist in a plasma with conditions similar to that of the solar corona. An avoided crossing is shown (see Figure 2(a)) to demonstrate that the different modes actually never cross and in fact take on the character of the mode that has been avoided. Abdelatif (1990) has shown that near these avoided crossings there is a coupling of the two different modes; they have a mixed character. With regards to dispersion, there are two distinct types of modes. One is the "slow" wave that is quite dispersive with the frequency staying fairly constant with increasing $\hat{k}_{x}$, apart from at avoided crossings. This is expected, as in a homogeneous and low plasma-beta medium the slow wave propagates mainly in the direction of the magnetic field. The other is a "fast" wave, mainly magnetic in nature, that is fairly non-dispersive for higher $k_{x}$ values.

It can be observed in Figure 2(b) that some eigenoscillations, in a plasma mimicking that of the photospheric/ chromospheric plasma layer, have periods between five and three minutes. One can also see that there are some modes that fall below the local Brunt-Väisäla frequency showing us that there is the possibility of " $g$-mode" type oscillations in this plasma. Note that the scales chosen for the dimensionless wavenumber in Figure 2(b), the waves are fairly dispersive, suggesting that waves are likely to be vertically propagating.

\section{TWO LAYER MODEL}

\subsection{Background}

Let us now consider a two-layer, gravitationally stratified plasma embedded in a uniform vertical magnetic field. The

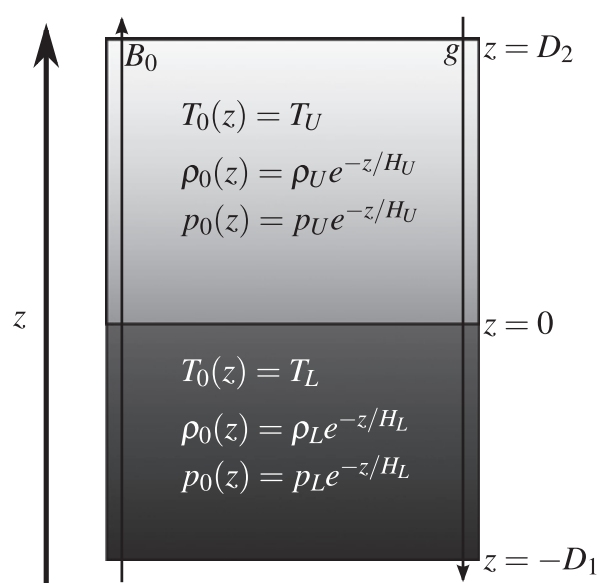

Figure 3. The background: a two-layer gravitationally stratified model of the global solar atmosphere.

ideal forms of the MHD equations are used i.e., Equations (3)(6). We also neglect the spherical shape of the Sun that gives an upper limit of the horizontal wavelength. The model is outlined in Figure 3. The lower layer is bounded by $z \in\left[-D_{1}, 0\right]$. The top layer is described within the bounds $z \in\left[0, D_{2}\right]$. Both layers are considered to be isothermal in the current approximation. There is a temperature discontinuity between the two media and, therefore, a density discontinuity. The lighter plasma is on top (upper) and the relatively heavy plasma in the bottom (lower). The plasma-beta $(\beta)$ is continuous across the discontinuity at $z=0$. From here on, we represent upper layer parameters with the subscript $U$ and lower layer parameters with the subscript $L$. We therefore introduce the quantity $\tau=T_{U} / T_{L}$, where $T_{U} / T_{L}$ is the isothermal temperature in the upper/lower layer. We, again, search for wave-like solutions. The aim is to find how global eigenmodes are affected by the jump in temperature across a discontinuity. Physically, the discontinuity could represent the thin "transition 
layer" or even the temperature increase from low chromosphere to high chromosphere. The background is assumed to be perturbed and we can therefore use the solutions derived previously. The solutions in the two separate layers are only different due to the difference in the parameters:

$$
\begin{aligned}
K_{U} & =K_{L} \tau, \quad \Omega_{U}=\Omega_{L} \tau, \quad \xi_{L}(z)=\frac{\omega H_{L}}{v_{A L}(0)} e^{-z / H_{L}}, \\
\xi_{U}(z) & =\frac{\omega H_{U}}{v_{A U}(0)} e^{-z / H_{U}}, \quad \frac{H_{U}}{H_{L}}=\tau,
\end{aligned}
$$

where,

$$
\begin{array}{ll}
K_{r}=k_{x} H_{r}, & \Omega_{r}=\frac{\omega H_{r} \sqrt{\gamma}}{v_{s r}}, \\
v_{s r}=\frac{\gamma p_{r}}{\rho_{r}}, & v_{A r}^{2}(0)=\frac{B_{0}^{2}}{2 \mu \rho_{r}},
\end{array}
$$

\subsection{Solutions and Dispersion Relation}

We look for global standing waves and, therefore, introduce the boundary conditions $v_{z}=v_{x}=0$ at $z=-D_{1}, D_{2}$. In the first model we study, the large density discontinuity is approximated as a perfectly reflective boundary. In the second model studied it is the solar wind flow speed that approximates the upper turning point, as it grows greater than the local Alfvén speed. The solutions must also be connected across the discontinuity. It can be shown that the consequences of the continuity conditions for the vertical magnetic field are that $v_{x}$, $v_{z}$, and their derivatives are continuous across the boundary at $z=0$. We define the velocity perturbations, $v_{x}$ and $v_{z}$ below:

$$
\begin{array}{ll}
v_{z}(z)=\sum_{j=1,5}^{4,8} C_{j} v_{z}^{(j)}(z), & v_{z}^{(j)}(z)=A_{j}^{(z)}, \\
v_{x}(z)=\sum_{j=1,5}^{4,8} C_{j} v_{x}^{(j)}(z), & v_{x}^{(j)}(z)=B_{j}^{(z)} .
\end{array}
$$

Note that when $j \in\{1,2,3,4\}$, all parameters are given by the values in the lower layer and when $j \in\{5,6,7,8\}$, all parameters are given by their corresponding values for the upper layer. The derivatives of $v_{x}$ and $v_{z}$ are denoted with a dash e.g., $A_{j}^{\prime(z)}, B_{j}^{\prime(z)}$. Evaluating $v_{x}, v_{z}$, and their derivatives using the boundary and continuity conditions, eight equations are obtained that can be cast in a matrix form as below,
For non-trivial solutions to this equation the determinant must be equal to zero. The determinant of the matrix on the left-hand side of Equation (30) is regarded from now on as a function of $\omega$ and $k_{x}$, say $\phi\left(\omega, k_{x}\right)$. We, therefore, look for the roots of $\phi(\omega$, $\left.k_{x}\right)=0$ numerically.

\subsubsection{Lower Solar Atmosphere (Model 1)}

The first model we focus on is analogous to the temperature jump from the photosphere/low chromosphere to the higher chromosphere. The lower boundary is the base of the photosphere/solar interior, while at the upper boundary there is a (large) drop in density and a large jump in temperature between the chromosphere and corona. Typical parameters for this model may be:

$$
\frac{D_{1}}{H_{L}}=8, \quad \frac{D_{2}}{H_{U}}=2,\left.\quad \beta\right|_{z=D 2}=0.1, \quad \tau=2 .
$$

For this model the dimensionless length $\hat{z}$ is used, where,

$$
\hat{z}=\frac{z}{D_{1}+D_{2}}, \quad \frac{D 1}{D_{1}+D_{2}}=\frac{2}{3}, \quad \frac{D_{2}}{D_{1}+D_{2}}=\frac{1}{3} .
$$

Figures 4(a), (b) show the numerical solutions to Equation (30) in the form of a dispersion diagram, with the frequency $(\hat{\omega})$ against the wavenumber $\left(\hat{k_{x}}\right)$. Indicated on the graphs are solutions where the oscillations correspond to five and three minute periods. The sound speed in the lower atmosphere is $7.9 \mathrm{~km} \mathrm{~s}^{-1}$ as before. One can identify from Figure 4(a) the so called "avoided crossings." An avoided crossing in this sense is where two separate solution paths start to meet, taking on characteristics of both solutions as they come closer together. These solution paths then literally avoid each other and carry on the trend of the other. To analyze these avoided crossings we refer to two excellent textbooks, i.e., Bray \& Loughhead (1974) and Goedbloed \& Poedts (2004). Using their formulations we now study the total wave energy density. The total wave energy density is comprised of the kinetic energy density (KE), magnetic energy density (ME), and the potential energy density (PE). The potential energy density can be split into gravitational potential (GE) and internal (IE). The total energy density is:

$$
E_{T}=\mathrm{KE}+\mathrm{ME}+\mathrm{IE}+\mathrm{GE},
$$

$$
\left(\begin{array}{cccccccc}
A_{1}^{(-D 1)} & A_{2}^{(-D 1)} & A_{3}^{(-D 1)} & A_{4}^{(-D 1)} & 0 & 0 & 0 & 0 \\
B_{1}^{(-D 1)} & B_{2}^{(-D 1)} & B_{3}^{(-D 1)} & B_{4}^{(-D 1)} & 0 & 0 & 0 & 0 \\
0 & 0 & 0 & 0 & A_{5}^{\left(D_{2}\right)} & A_{6}^{\left(D_{2}\right)} & A_{7}^{\left(D_{2}\right)} & A_{8}^{\left(D_{2}\right)} \\
0 & 0 & 0 & 0 & B_{5}^{\left(D_{2}\right)} & B_{6}^{\left(D_{2}\right)} & B_{7}^{\left(D_{2}\right)} & B_{8}^{\left(D_{2}\right)} \\
A_{1}^{(0)} & A_{2}^{(0)} & A_{3}^{(0)} & A_{4}^{(0)} & -A_{5}^{(0)} & -A_{6}^{(0)} & -A_{7}^{(0)} & -A_{8}^{(0)} \\
B_{1}^{(0)} & B_{2}^{(0)} & B_{3}^{(0)} & B_{4}^{(0)} & -B_{5}^{(0)} & -B_{6}^{(0)} & -B_{7}^{(0)} & -B_{8}^{(0)} \\
A_{1}^{\prime(0)} & A_{2}^{\prime(0)} & A_{3}^{\prime(0)} & A_{4}^{\prime(0)} & -A_{5}^{\prime(0)} & -A_{6}^{\prime(0)} & -A_{7}^{\prime(0)} & -A_{8}^{\prime(0)} \\
B_{1}^{\prime(0)} & B_{2}^{\prime(0)} & B_{3}^{\prime(0)} & B_{4}^{\prime(0)} & -B_{5}^{\prime(0)} & -B_{6}^{\prime(0)} & -B_{7}^{\prime(0)} & -B_{8}^{\prime(0)}
\end{array}\right)\left(\begin{array}{l}
C_{1} \\
C_{2} \\
C_{3} \\
C_{4} \\
C_{5} \\
C_{6} \\
C_{7} \\
C_{8}
\end{array}\right)=0 .
$$




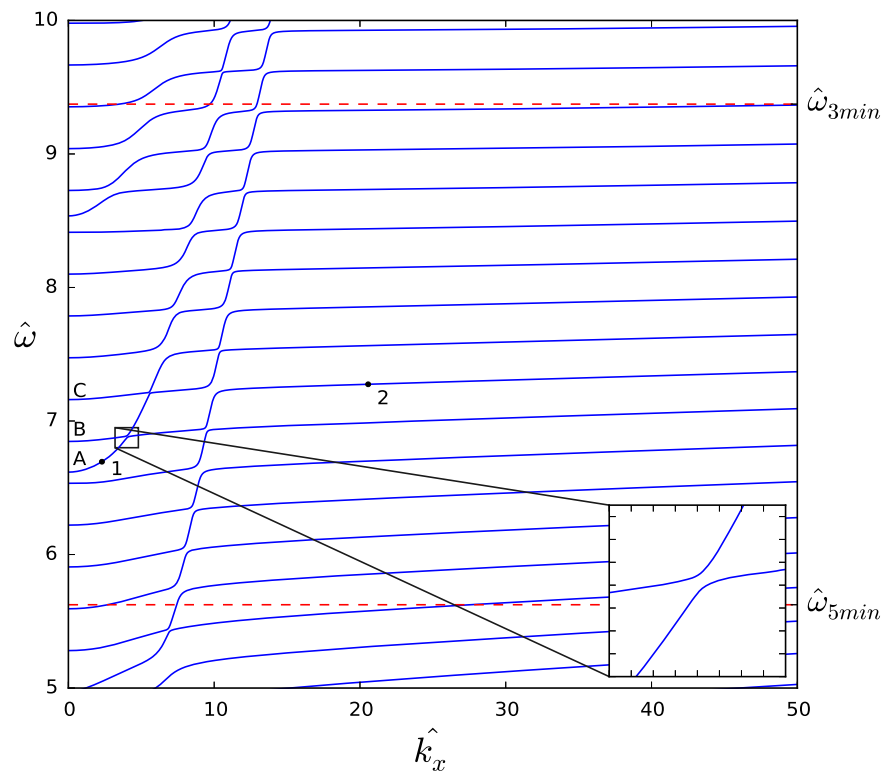

(a)

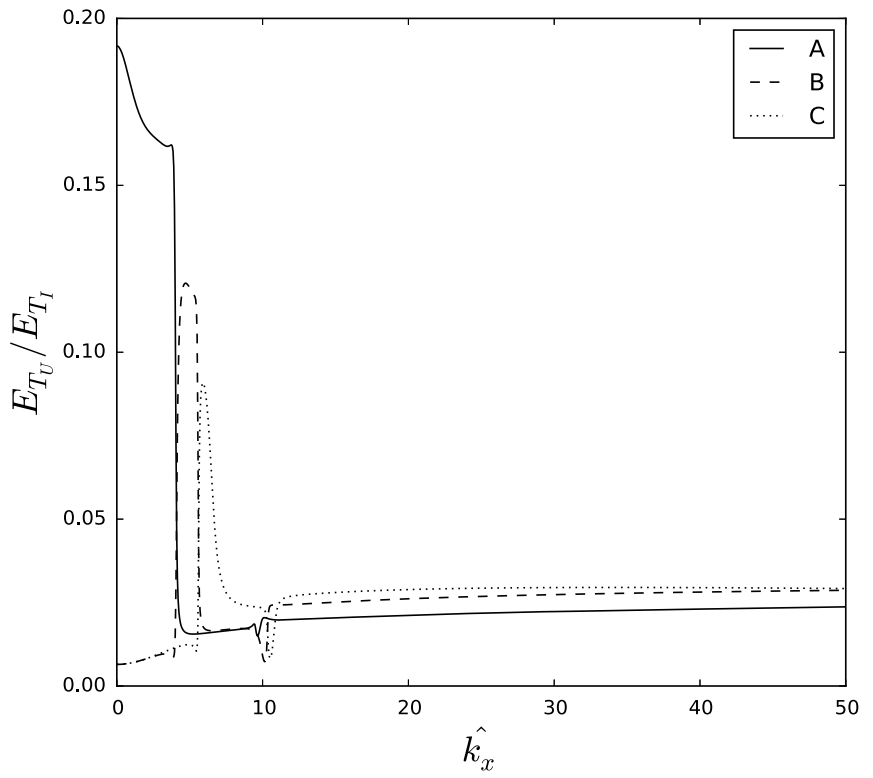

(b)

Figure 4. (a) The dispersion relation for a model of the lower solar atmosphere, with frequency plotted against wavenumber. The lines $\hat{\omega}_{3 \text { min }}$ and $\hat{\omega}_{5}$ min are dashed lines corresponding to a lower layer sound speed of $7.9 \mathrm{~km} \mathrm{~s}^{-1}$. An avoided crossing is extracted to illustrate that the eigen-frequency solutions do not cross here. (b) The total wave energy distribution, as described by the equation for the lines A, B, C in (a) for varying $\hat{k_{x}}$. Points 1 and 2 of Figure 4 are of special interest, see e.g., Figures 5-7.

where,

$$
\begin{aligned}
& \mathrm{KE}=\frac{1}{2} \rho_{0}\left(v_{x}^{2}+v_{z}^{2}\right), \quad \mathrm{IE}=\frac{p_{1}^{2}}{2 \rho_{0} C_{s}^{2}}, \\
& \mathrm{GE}=\frac{\rho_{0} N^{2} v_{z}^{2}}{2 \omega^{2}}, \quad \mathrm{ME}=\frac{1}{2 \mu}\left(B_{x}^{2}+B_{z}^{2}\right) .
\end{aligned}
$$

Abdelatif (1990) studied the components of the wave energy density for a bounded one-layer plasma. In the study it is shown that, in the vicinity of the avoided crossings, as the two modes (solutions paths on the $\hat{\omega}-\hat{k}_{x}$ diagram) approach one another, both have shared characteristics i.e., the distributions of the components of the wave energy density are very similar. As the solution paths then diverge, one solution takes on the characteristics of the other and vice-versa. To study the wave energy density distribution in each layer, the integral of the total wave energy density over the whole cavity and the integral of the total wave energy density in the upper layer are introduced:

$$
\begin{aligned}
& E_{T_{I}}=\int_{-D_{1}}^{D_{2}} E_{T}\left(z, \hat{\omega}, \hat{k_{x}}\right) d z \\
& E_{T_{U}}=\int_{0}^{D_{2}} E_{U}\left(z, \hat{\omega}, \hat{k_{x}}\right) d z, \quad E_{D}=\frac{E_{T_{U}}}{E_{T_{I}}}, \\
& E_{T}\left(z, \hat{\omega}, \hat{k_{x}}\right)=\left\{\begin{array}{l}
E_{L}\left(z, \hat{\omega}, \hat{k_{x}}\right) \quad z \leqslant 0 \\
E_{U}\left(z, \hat{\omega}, \hat{k_{x}}\right) \quad z \geqslant 0
\end{array}\right\} .
\end{aligned}
$$

Here, $E_{T_{I}}$ is the total wave energy density integrated across both layers. $E_{T}$ is the total wave energy density as a function of height, frequency, and horizontal wavenumber and $E_{T_{U}}$ is the total wave energy density integrated from $z=0$ to $z=D_{2}$.

Figure 4(b) shows the change in wave energy density for the solutions A, B, and C of Figure 4(a). The wave energy density is mostly confined to the lower layer, as the maximum value of $E_{T_{U}} / E_{T_{I}}$ is around 0.20. Again, at the avoided crossings, the modes take on the characteristics of the mode they have just avoided. This can be seen, for example, as the curves A and B approach and then separate, a drop in $E_{T_{U}} / E_{T_{I}}$ for $\mathrm{A}$ and an increase for $\mathrm{B}$, respectively, can be noted.

Figure 5(a) shows the wave energy density components for point one on Figure 4(a). It can be seen that the wave is mainly of acoustic nature but also has some magnetic characteristics. As there is a changing plasma-beta (going from high to low with an increase in height) it is difficult to class the mode as "slow" or "fast." This is due to the mode being practically of acoustic nature and would therefore be classed as "fast" at $z=-D 1$ (high-beta) but slow at $z=D_{2}$ (low-beta). The magnetic component of the wave is found to drop off dramatically with increasing height in the atmosphere and the wave becomes primarily an acoustic wave. At the discontinuity the wave energy density drops almost by a factor of 2 , but there is still a significant portion trapped in the upper layer.

Figure 5(b) shows the wave energy density components of point two on Figure 4(a). The standing wave at the bottom of the atmosphere is of mainly magnetic nature in the high-beta atmosphere. The wave energy density drops off dramatically with height. This is due to the change of Alfvén speed, which is increasing considerably with height, and therefore most of the wave's energy density is reflected before the discontinuity is reached.

\subsubsection{Lower Solar Atmosphere to Corona (Model 2)}

The second model is analogous to a temperature jump from the lower atmosphere to the corona. The upper turning point is, physically, considered to be when the solar wind flow speed growth is greater than the local Alfvén speed. The parameters 


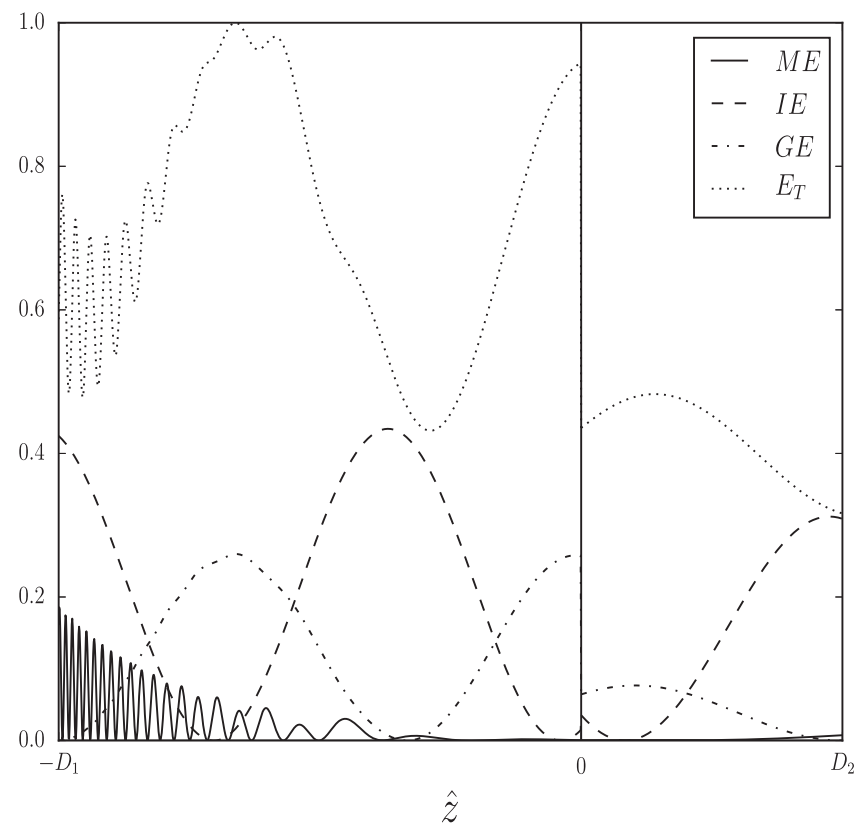

(a)

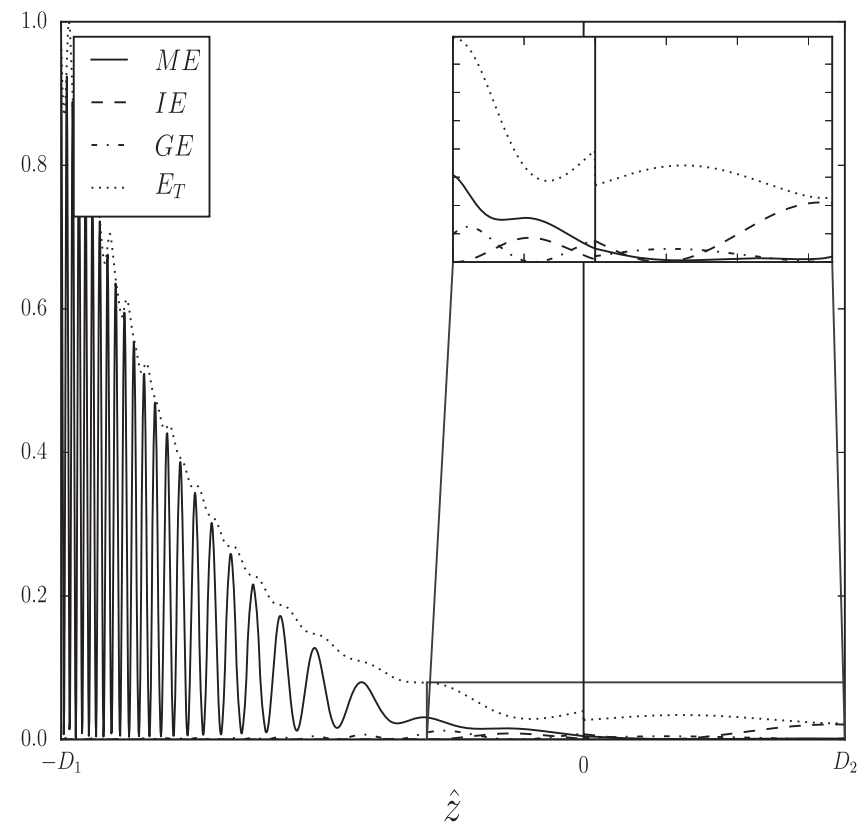

(b)

Figure 5. (a) The components of the wave energy for point one in Figure 4 (a). $\hat{\omega}=6.695, \hat{k_{x}}=2.302$. (b) The components of the wave energy for point two of Figure 4 (a). $\hat{\omega}=7.275, \hat{k_{x}}=20.563$. Note that the kinetic energy has been left out of these diagrams and are shown in Figures 6(b) and 7(b), respectively. Figure 5(b) shows a zoomed in portion within the box in the uppermost right corner of the figure.

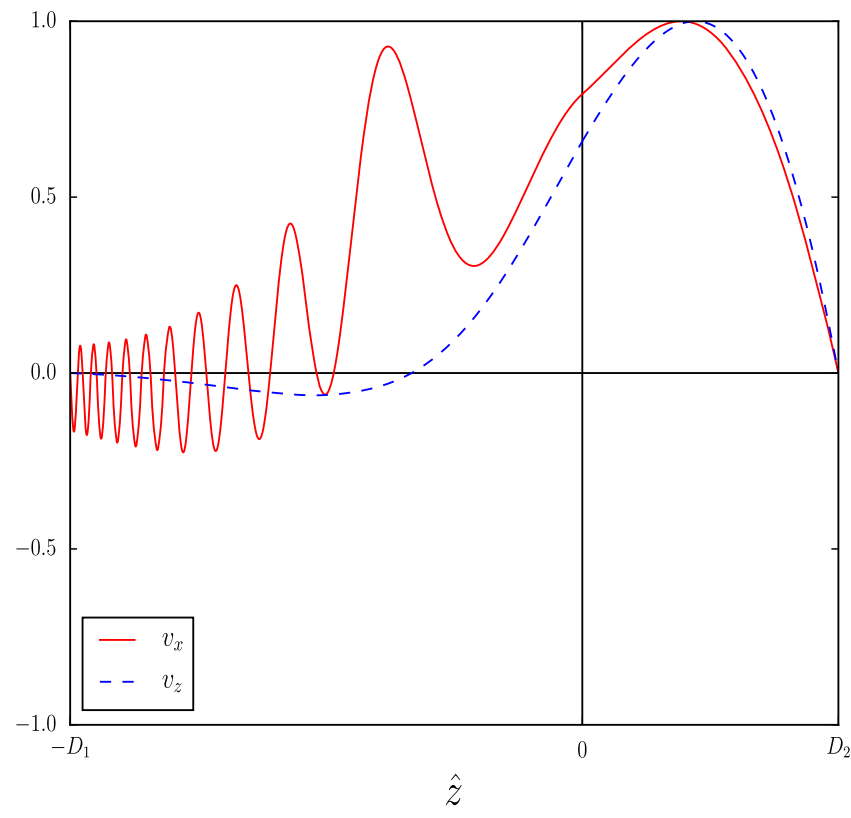

(a)

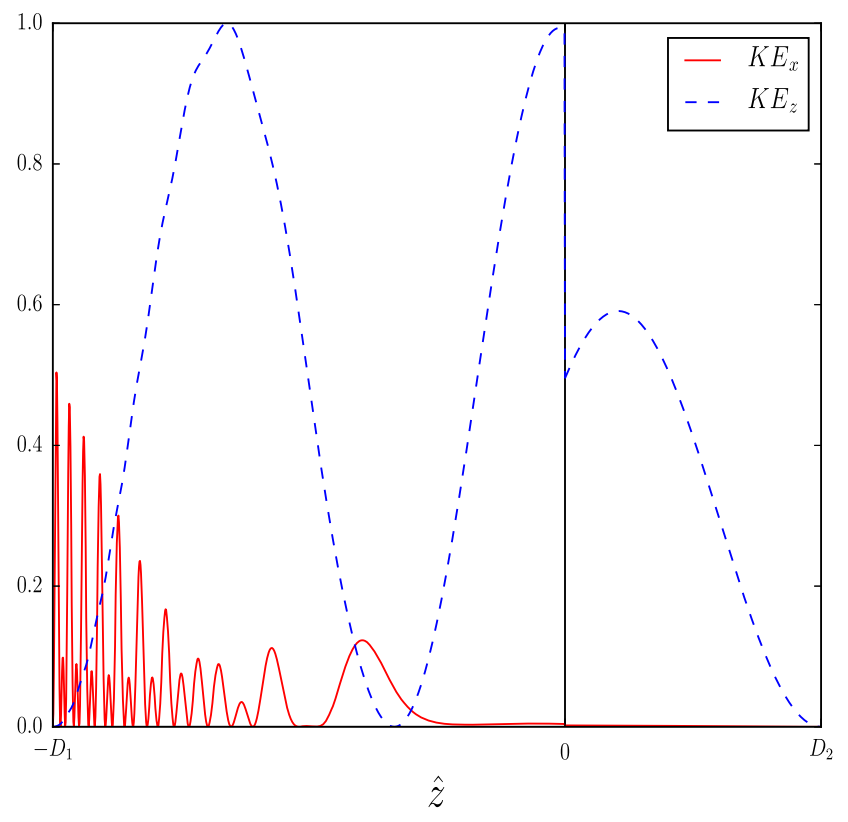

(b)

Figure 6. (a) The eigenfunctions of point one in Figure 4(a), with $\hat{\omega}=6.695, \hat{k_{x}}=2.302$. (b) The kinetic energy density in the $x$ - and $z$-directions (i.e., KEx, and KE $z$, respectively) of point one in Figure 4(a).

for this model are given below:

$$
\frac{D_{1}}{H_{L}}=7, \quad \frac{D_{2}}{H_{U}}=3,\left.\quad \beta\right|_{z=0}=0.1, \quad \tau=400 .
$$

For this model the dimensionless length $\hat{z}$ is used, where,

$$
\hat{z}=\frac{z}{D_{1}+D_{2}}, \quad \frac{D 1}{D_{1}+D_{2}}=\frac{7}{1207}, \quad \frac{D_{2}}{D_{1}+D_{2}}=\frac{1200}{1207} .
$$




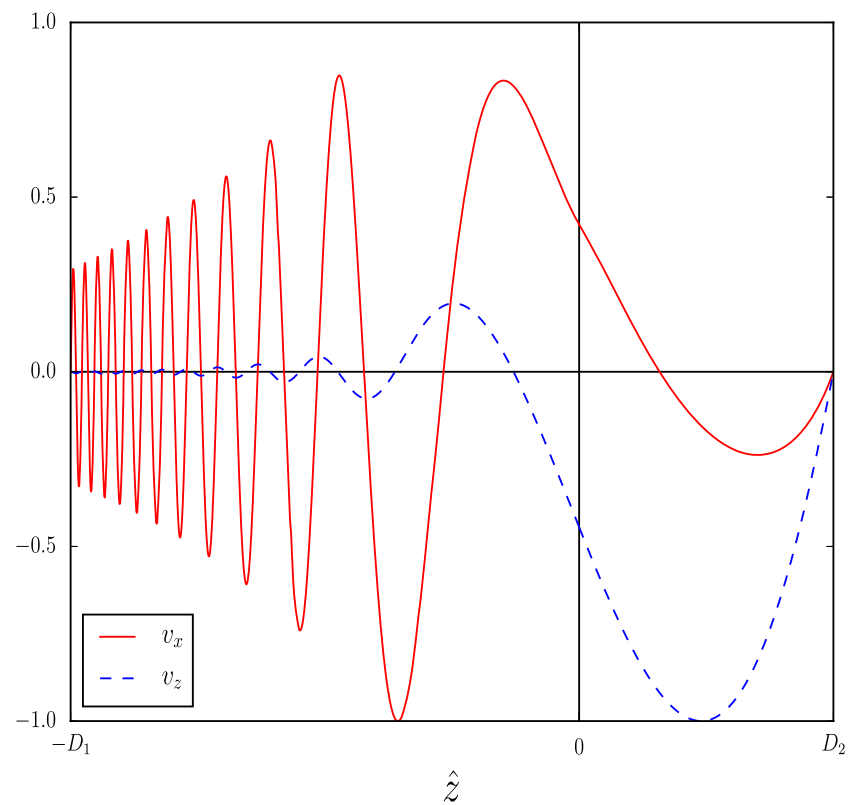

(a)

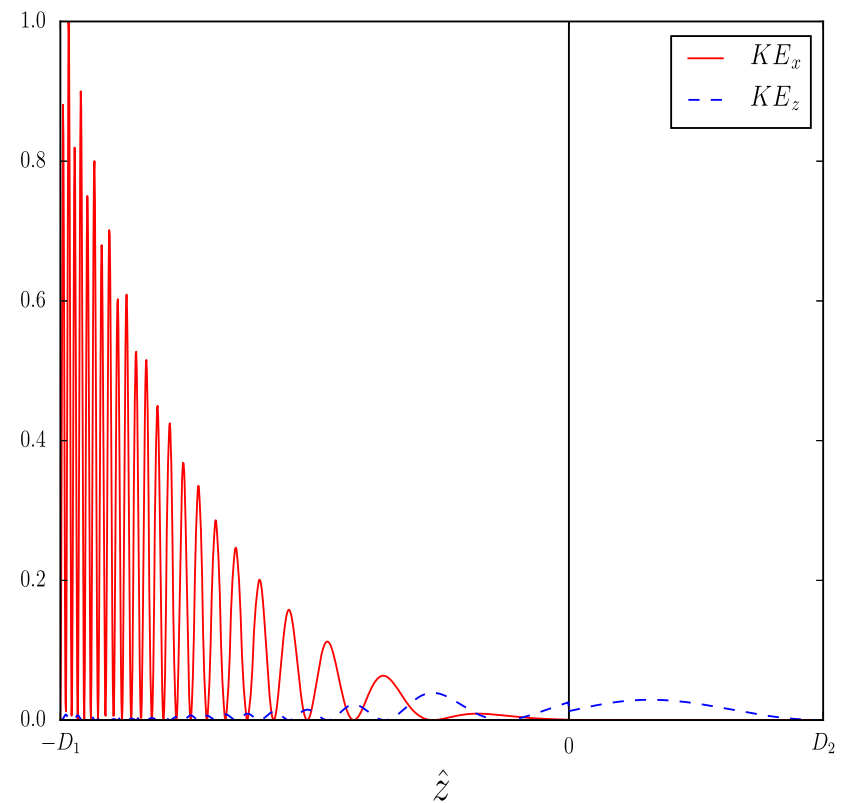

(b)

Figure 7. Same as Figure 6 for point two in Figure 4(a), with $\hat{\omega}=7.275, \hat{k_{x}}=20.563$.
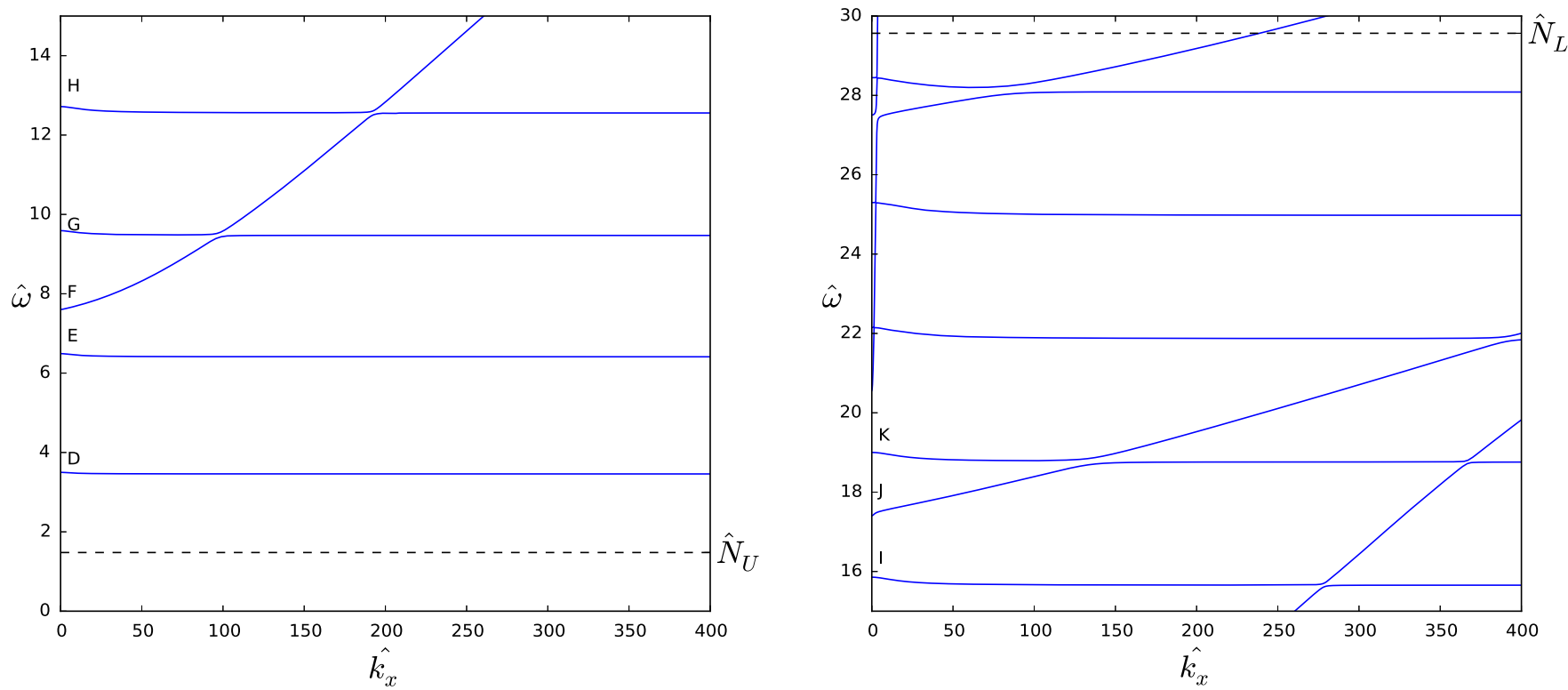

Figure 8. The dispersion relation for the lower atmosphere to corona model, with frequency plotted against wavenumber. Noted are the Brunt-Väisäla frequencies for the upper and lower layers, respectively. On the left are the eigenmodes up to $\hat{\omega}=15$, while on the right are the eigenmodes from $\hat{\omega}=15$ to $\hat{\omega}=30$.

Figure 8 graphs the dispersion curves for this model, for dimensionless frequencies ranging between $\hat{\omega}=0$ to $\hat{\omega}=30$ with $\hat{k_{x}}$ between 0 and 400. Figure 9 shows the same case but between $\hat{\omega}=0$ to $\hat{\omega}=30$ instead, and with the dispersion curves for the separate single layer models, as a comparison. One can see from Figures 9(a)-(d), as expected, that the large density discontinuity acts as a physical barrier that reflects practically all of the wave's energy density. This is evidenced in both Figures 9(c) and (d) where the energy density is mainly trapped in the upper or lower layers but at an avoided crossing the nature of each mode switches. The modes are inherently coupled together. The dispersion relation is therefore rather similar to that of a single-layer plasma. There are, however, avoided crossings between the solutions in the upper layer and bottom layer. It is also possible to see from Figures 9(a) and (b) that the lower layer solutions are frequency shifted slightly for $\hat{k_{x}}$ up to about 200 which corresponds to a small $K_{L}$ value, the discontinuity seems to lower the frequency of these modes slightly. It is possible to find an approximation for this frequency shift analytically for $\hat{k_{x}}=0$. Using the solutions of Equations (10) and (11) and applying the boundary conditions as described before, two distinct dispersion relations can be found for $v_{x}$ and $v_{z}$ respectively. The dispersion relation for $v_{x}$ is highly transcendental, and analytic study is, to our best 


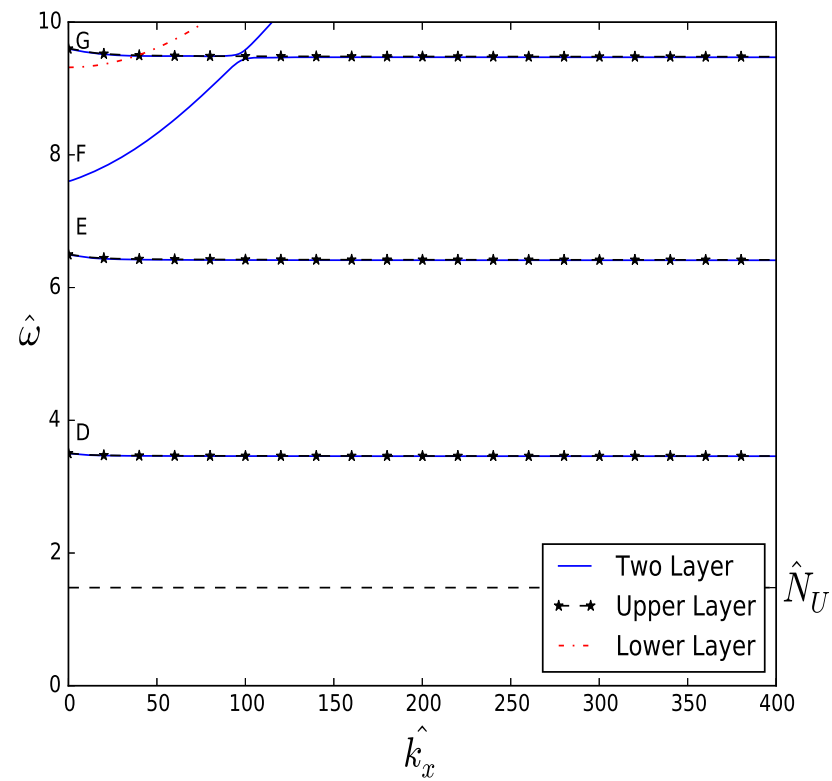

(a)

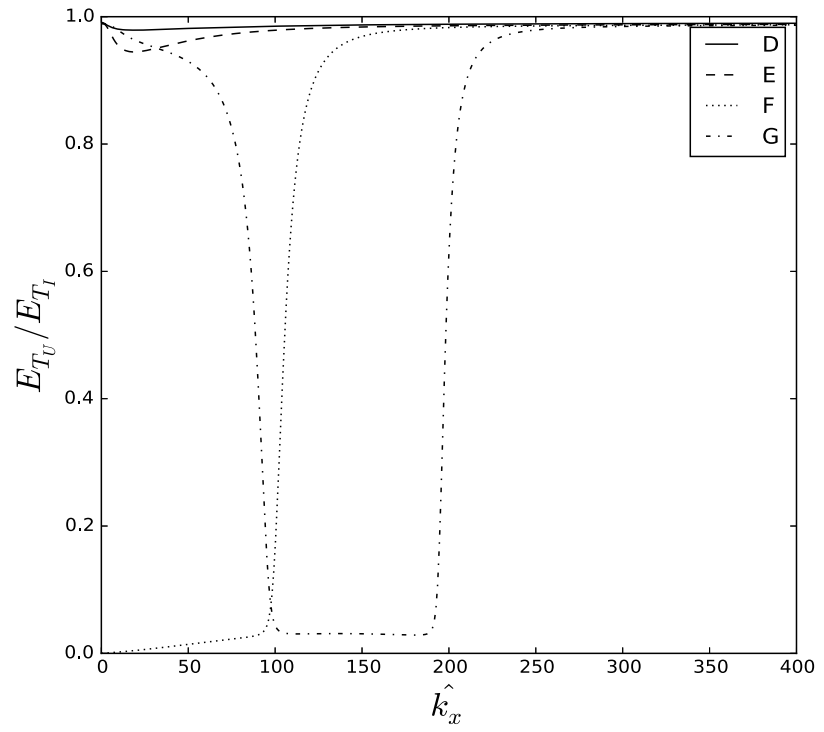

(c)

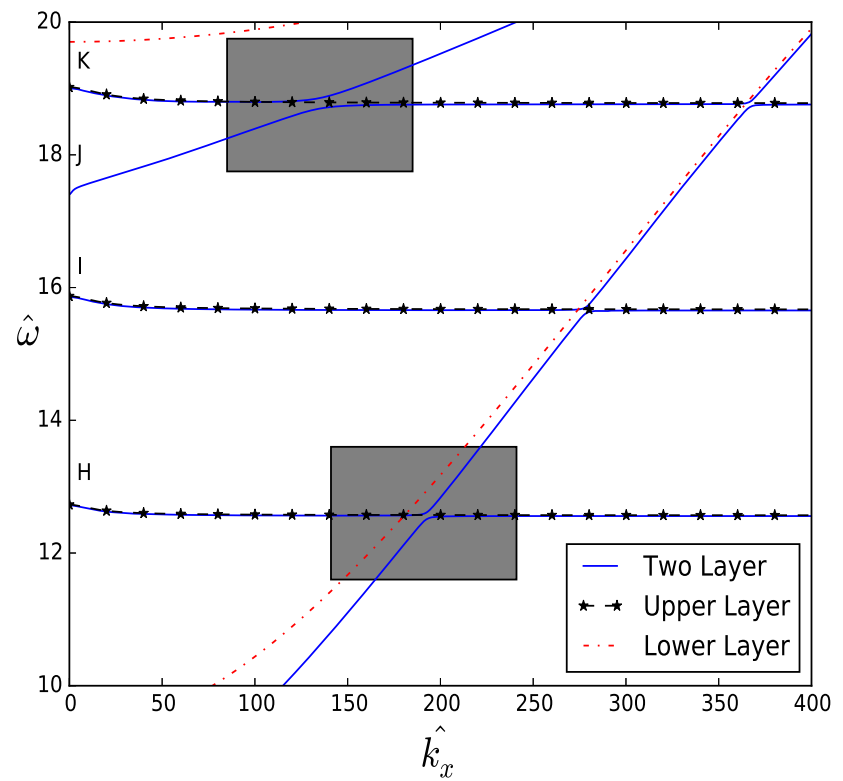

(b)

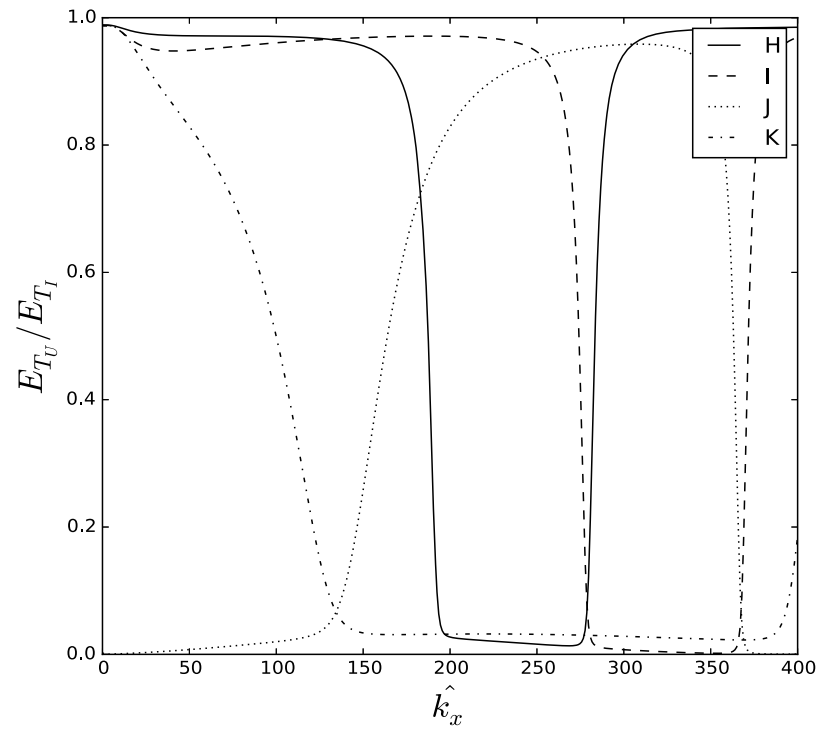

(d)

Figure 9. Figures 9(a) and (b) (upper left/right) show the first eight eigenmodes of model (1) with the single-layer atmospheres (upper and lower) laid over (Figure 9(b) is a continuation of Figure 9(a)). The shaded boxes in Figure 9(b) are used to emphasize two avoided crossings between the upper and lower layer eigenmodes. Figures 9(c) and (d) (lower left/right) show the corresponding energy density distribution of these modes (whether their energy density is in the upper or lower layer) with a varying wavenumber $\left(\hat{k_{x}}\right)$. The letters $D, \ldots, K$ label the lines.

knowledge, not possible. However, the dispersion relation for $v_{z}$ is much more malleable and is presented below:

$$
\begin{aligned}
& \sin \left(q_{0 L} \hat{D_{1}}\right) \sin \left(q_{0 U} \hat{D_{2}}\right)\left(1-\frac{1}{\tau}\right) \\
& \quad+2 \sin \left(q_{0 L} \hat{D_{1}}\right) \cos \left(q_{0 U} \hat{D_{2}}\right) \frac{q_{0 U}}{\tau} \\
& \quad+2 \sin \left(q_{0 U} \hat{D_{2}}\right) \cos \left(q_{0 L} \hat{D_{1}}\right) q_{0 L}=0 .
\end{aligned}
$$

Here, $\hat{D_{1}}=D_{1} / H_{L}$ and $\hat{D_{2}}=D_{2} / H_{U}$. Due to the very large temperature increase between the two layers, $\tau$ is a very large number so that the approximation, $\tau^{1 / 2} \gg 1$, can be made. Therefore any terms of order $(1 / \tau)^{1 / 2}$ or higher can be neglected, resulting in the following dispersion relation

$$
\sin \left(q_{0 U} \hat{D_{2}}\right)\left(\tan \left(q_{0 L} \hat{D_{1}}\right)+2 q_{0 L}\right) \approx 0 .
$$

The first term just refers to sound waves trapped in the upper layer, reflected by the temperature discontinuity. The second term refers to sound waves in the lower layer with a correction due to the discontinuity. If we denote $\delta=2 / \hat{D}_{1}$ and suggest that this is a small quantity, it is possible to use a perturbation method to find an approximation of the frequencies

$$
\hat{\omega}^{2} \approx \frac{\left(\hat{D_{1}}+\hat{D_{2}} \tau\right)^{2}}{\tau}\left(\frac{1}{4}+\frac{m^{2} \pi^{2}}{{\hat{D_{1}}}^{2}}\left(1-\delta+\delta^{2}+O\left(\delta^{3}\right)\right)^{2}\right) .
$$


Taking the lowest order approximation in $\delta$ of Equation (35), it can be seen that the frequencies are slightly lower when compared to that of the single layer (SL) which is shown below as a comparison

$$
\hat{\omega}_{\mathrm{SL}}^{2}=\frac{\left(\hat{D_{1}}+\hat{D_{2}} \tau\right)^{2}}{\tau}\left(\frac{1}{4}+\frac{m^{2} \pi^{2}}{{\hat{D_{1}}}^{2}}\right) .
$$

Therefore, to the lowest order in $\delta$, the frequency shift, $\Delta \hat{\omega}^{2}$, is given by

$$
\Delta \hat{\omega}^{2}=-4 \frac{\left(\hat{D_{1}}+\hat{D_{2}} \tau\right)^{2}}{\tau} \frac{m^{2} \pi^{2}}{\hat{D}_{1}{ }^{3}} .
$$

This result agrees with those plotted Figures 9(a) and (b) that the frequency of the lower layer is reduced (for small wavenumbers) due to having some continuity across the layer, while the frequency of the upper layer solution seems to be almost identical. Physically, this decrease in frequency is, we suggest, due to the fact that when the standing wave is set up a small amount of time is taken for the wave to travel in the upper layer and reflect back.

\section{CONCLUSION}

The main focus of this work is to show analytic insight into a stratified, isothermal plasma with a "vertical" magnetic field. Analytic solutions are important as they help with understanding the physics of a model compared to solving the equations. Meijer-G functions were found as solutions for a vertical field, along with hypergeometric functions for the horizontal velocity component, $v_{x}$, (see also Zhugzhda 1979). Leroy \& Schwartz (1982) presented Fröbenius series solutions for $v_{x}$ and $v_{z}$.

We have been able to derive a governing equation for a new variable, $\Theta=p_{1} / \rho_{0}$, which is, in fact, the scaled dimensionless pressure, $p_{1} / p_{0}$, due to the isothermal nature of the background plasma. The variable $\Theta$ was found in terms of hypergeometric functions as a solution of Equation (16). From these solutions it was fairly simple to confirm the hypergeometric solutions for $v_{x}$, given by Zhugzhda (1979). However, the first most notable result was the derivation of the solutions for $v_{z}$ in terms of four linearly independent hypergeometric functions (for most cases). These solutions are noted to be exactly the same as the Fröbenius solution derived by Leroy \& Schwartz (1982). It was never realized until now that these could be more simply written as ${ }_{3} F_{4}$ hypergeometric functions. Next, the limit of a small wavelength was taken, i.e., $k_{x} \rightarrow \infty$. The dispersion relation in this limit was relatively straightforward to derive and the necessary approximations of the hypergeometric functions were found as well. Taking the high-beta limit, the dispersion relation confirmed the result in Hasan \& ChristensenDalsgaard (1992).

The next part of the investigation was to consider a two-layer bounded plasma, with the intention to make a first-insight application to the global solar atmosphere. A two-layer model was suggested by Yelles Chaouche \& Abdelatif (2005) but with an upper boundary condition of finite energy density as $z \rightarrow \infty$. The wavenumber was found to have an imaginary component. In a two-layer bounded atmosphere this was not seen to occur. Two different model atmospheres were considered. The first was analogous to the temperature jump between the photosphere/low chromosphere to the high chromosphere. Eigen-frequencies were found within the band of three and five minute oscillations for viable physical parameters. The larger the wavenumber, $\hat{k_{x}}$, the less energy density of the wave was contained in the upper layer. The energy of these so called "magnetic" modes was found to decrease rapidly with height. This is physically caused by the change in the Alfvén speed with height and therefore the wave is reflected. However, it was shown for certain modes e.g., as a typical example, when $\hat{\omega}=6.695$ and $\hat{k_{x}}=2.302$, an "acoustic-gravity" mode was seen to share wave energy density relatively equally between the lower atmosphere and the upper atmosphere (with a small drop in total wave energy density across the discontinuity). This distribution of wave energy density, along with dissipative processes, could lead to heating in the higher solar atmosphere.

The second model considered a transition in temperature reminiscent of that between the lower solar atmosphere and the corona; a factor of 400 increase in temperature was implemented in the equilibrium. It was found that solutions to the dispersion relation were practically those of the upper and lower single-layer atmospheres. Physically, this just shows that the large density discontinuity acts as a physical barrier to reflect waves incident on this surface. However, it was also found that around the avoided crossings there would always be some inherent coupling where the solutions change character between the upper and lower layer solutions, with the transition sometimes smooth. This coupling is important as it allows wave energy to leak from one layer to another. Given the huge change (i.e., drop) in inertia of going from the chromosphere to the corona, even a small leakage may have considerable effects on the oscillations in the corona.

Further work could be implemented for a three-layer atmosphere to model the transition of the global modes between the photosphere/low chromosphere, high chromosphere, and corona. Another exciting direction may be to investigate mode conversion, well beyond the scope of the current focus.

This work is supported by the UK Science and Technology Facilities Council (STFC). The authors would like to acknowledge the SunPy, NumPy, SciPy, Matplotlib, and MpMath python projects for providing computational tools. R.E. acknowledges the support received from the Royal Society (UK) and from NSF (Hungary), OTKA K83133. The authors would like to thank the referee for the very useful comments on the work.

\section{APPENDIX}

Three equations are obtained from the linearized MHD equations relating $\Theta, v_{x}$, and $v_{z}$,

$$
\begin{gathered}
k_{x} \omega \Theta=v_{a}^{2} v_{x}^{\prime \prime}+\left(\omega^{2}-v_{a}^{2} k_{x}^{2}\right) v_{x} . \\
v_{s}^{2}\left(\omega^{2}-N^{2}\right) k_{x} v_{x}=\omega\left(v_{s}^{2} \Theta^{\prime \prime}-\frac{v_{s}^{2}}{H} \Theta^{\prime}+\omega^{2} \Theta\right) . \\
\left(\omega^{2}-N^{2}\right) v_{z}=-i \omega\left(\Theta^{\prime}-\frac{N^{2}}{g} \Theta\right),
\end{gathered}
$$

where $N=\left(\frac{(\gamma-1) g^{2}}{v_{s}^{2}}\right)^{1 / 2}$ is the Brunt-Väisälla frequency in an isothermal plasma. Equations (38) and (39) permit a fourth 
order differential equation for $\Theta$ to be found:

$$
\begin{aligned}
& v_{s}^{2} v_{a}^{2} \Theta^{\prime \prime \prime \prime}-\frac{v_{a}^{2} v_{s}^{2}}{H} \Theta^{\prime \prime \prime}+\left[\left(v_{a}^{2}+v_{s}^{2}\right) \omega^{2}-k_{x}^{2} v_{a}^{2} v_{s}^{2}\right] \Theta^{\prime \prime} \\
& -\frac{v_{s}^{2}}{H}\left[\omega^{2}-v_{a}^{2} k_{x}^{2}\right] \Theta^{\prime}+\left[\omega^{4}-k_{x}^{2}\left(v_{a}^{2}+v_{s}^{2}\right) \omega^{2}\right. \\
& \left.+k_{x}^{2} v_{s}^{2} N^{2}\right] \Theta=0 .
\end{aligned}
$$

Introducing again the dimensionless variables,

$$
\xi=\frac{\omega H}{v_{a}}, \quad \Omega=\frac{\omega H \sqrt{\gamma}}{v_{s}}, \quad K=k_{x} H,
$$

Equation (41) can be transformed, with $\xi$ as a new independent variable, to:

$$
\begin{aligned}
& \xi^{4} \frac{d^{4} \Theta}{d \xi^{4}}+8 \xi^{3} \frac{d^{3} \Theta}{d \xi^{3}}+\xi^{2}\left[4 \xi^{2}+13+4\left(\frac{\Omega^{2}}{\gamma}-K^{2}\right)\right] \frac{d^{2} \Theta}{d \xi^{2}} \\
& +\xi\left[12 \xi^{2}+3+4 \frac{\Omega^{2}}{\gamma}-12 K^{2}\right] \frac{d \Theta}{d \xi} \\
& \quad+16\left[\left(\frac{\Omega^{2}}{\gamma}+K^{2}\left(\frac{\gamma-1}{\gamma \Omega^{2}}-1\right)\right) \xi^{2}-\frac{K^{2} \Omega^{2}}{\gamma}\right] \Theta \\
& =0 .
\end{aligned}
$$

Solutions to Equation (16) can be found, using the Fröbenius method, as series solutions. These series solutions can then be written as hypergeometric functions:

$$
\begin{aligned}
& \Theta=C_{1} \xi^{2 K_{2} F_{3}}
\end{aligned}
$$

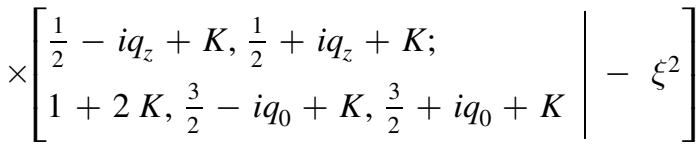

$$
\begin{aligned}
& +C_{2} \xi^{-2 K}{ }_{2} F_{3}\left[\begin{array}{l}
\frac{1}{2}+i q_{z}-K, \frac{1}{2}-i q_{z}-K \\
1-2 K, \frac{3}{2}-i q_{0}-K, \frac{3}{2}+i q_{0}-K
\end{array} \mid-\xi^{2}\right] \\
& +C_{3} \xi^{-1+i 2 q_{0}}{ }_{2} F_{3}
\end{aligned}
$$

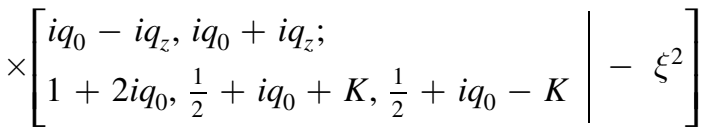

$$
\begin{aligned}
& +C_{4} \xi^{-1-i 2 q_{0}}{ }_{2} F_{3} \\
& \times\left[\begin{array}{l}
-i q_{z}-i q_{0}, i q_{z}-i q_{0} ; \\
1-2 i q_{0}, \frac{1}{2}-i q_{0}+K, \frac{1}{2}-i q_{0}-K
\end{array} \mid-\xi^{2}\right] .
\end{aligned}
$$

From now on the hypergeometric functions are written in the following form, for ease of use,

$$
\begin{aligned}
& F_{3}\left[\begin{array}{c|c}
\frac{1}{2}-i q_{z}+K, \frac{1}{2}+i q_{z}+K \\
1+2 K, \frac{3}{2}-i q_{0}+K, \frac{3}{2}+i q_{0}+K & -\xi^{2}
\end{array}\right] \\
& ={ }_{2} F_{3}\left[\begin{array}{c}
a_{11}, a_{12} ; \\
b_{11}, b_{12}, b_{13}
\end{array} \mid-\xi^{2}\right],
\end{aligned}
$$

$$
\begin{aligned}
& F_{3}\left[\begin{array}{c}
\frac{1}{2}+i q_{z}-K, \frac{1}{2}-i q_{z}-K ; \\
1-2 K, \frac{3}{2}-i q_{0}-K, \frac{3}{2}+i q_{0}-K
\end{array}\right] \\
& \quad={ }_{2} F_{3}\left[\begin{array}{c}
a_{21}, a_{22} ; \\
b_{21}, b_{22}, b_{23}
\end{array} \mid-\xi^{2}\right], \\
& F_{3}\left[\begin{array}{c}
i q_{0}-i q_{z}, i q_{0}+i q_{z} ; \\
1+2 i q_{0}, \frac{1}{2}+i q_{0}+K, \frac{1}{2}+i q_{0}-K
\end{array}\right] \\
& \quad={ }_{2} F_{3}\left[\begin{array}{c}
a_{31}, a_{32} ; \\
b_{31}, b_{32}, b_{33}
\end{array} \mid-\xi^{2}\right], \\
& F_{3}\left[\begin{array}{c}
-i q_{z}-i q_{0}, i q_{z}-i q_{0} ; \\
1-2 i q_{0}, \frac{1}{2}-i q_{0}+K, \frac{1}{2}-i q_{0}-K \mid
\end{array}\right] \\
& ={ }_{2} F_{3}\left[\begin{array}{c}
a_{41}, a_{42} ; \\
b_{41}, b_{42}, b_{43}
\end{array} \mid-\xi^{2}\right] .
\end{aligned}
$$

The derivation of the solutions of $v_{x}$ and $v_{z}$ follows below. Denoting from now on,

$$
\Theta^{\prime}:=\frac{d \Theta}{d \xi^{2}}
$$

The derivative (with respect to $\xi^{2}$ ) of an arbitrary hypergeometric function $\left({ }_{2} F_{3}\right)$ multiplied by a power function is given as:

$$
\begin{gathered}
\frac{d}{d \xi^{2}}\left(\xi_{j}^{\mu} \sum_{n=0}^{\infty} \frac{(a)_{n}(b)_{n}\left(-\xi^{2}\right)^{n}}{(c)_{n}(d)_{n}(e)_{n} n !}\right) \\
=\xi^{\mu_{j}-2} \sum_{n=0}^{\infty} \frac{(a)_{n}(b)_{n}\left(-\xi^{2}\right)^{n}}{(c)_{n}(d)_{n}(e)_{n} n !}\left(\frac{\mu_{j}}{2}+n\right), \\
\frac{d^{2}}{d\left(\xi^{2}\right)^{2}}\left(\xi^{\mu_{j}} \sum_{n=0}^{\infty} \frac{(a)_{n}(b)_{n}\left(-\xi^{2}\right)^{n}}{(c)_{n}(d)_{n}(e)_{n} n !}\right) \\
=\xi^{\mu_{j}-4} \sum_{n=0}^{\infty} \frac{(a)_{n}(b)_{n}\left(-\xi^{2}\right)^{n}}{(c)_{n}(d)_{n}(e)_{n} n !}\left(\frac{\mu_{j}}{2}+n\right)\left(\frac{\mu_{j}}{2}+n-1\right),
\end{gathered}
$$

where $j=1,2,3$, or 4 and

$$
\begin{aligned}
& \mu_{1}=2 K, \quad \mu_{2}=-2 K, \\
& \mu_{3}=-1+i 2 q_{0}, \quad \mu_{4}=-1-i 2 q_{0} .
\end{aligned}
$$

The derivatives of $z$ can be written in terms of derivatives of $\xi^{2}$,

$$
\frac{d}{d z}=\frac{\partial \xi^{2}}{\partial z} \frac{d}{d \xi^{2}}=-\frac{\xi^{2}}{H} \frac{d}{d \xi^{2}}, \quad \frac{d^{2}}{d z^{2}}=\frac{\xi^{2}}{H^{2}} \frac{d}{d \xi^{2}}+\frac{\xi^{4}}{H^{2}} \frac{d^{2}}{d\left(\xi^{2}\right)^{2}} .
$$

Equation (39) shows $v_{x}$ in terms of derivatives of $\Theta$. Writing this in terms of derivatives with respect to $\xi^{2}$ and some dimensionless variables,

$$
v_{x}=\frac{\omega}{k_{x}\left(\omega^{2}-N^{2}\right)} \frac{1}{H^{2}}\left(\xi^{4} \Theta^{\prime \prime}+2 \xi^{2} \Theta^{\prime}+\frac{\Omega^{2}}{\gamma} \Theta\right) .
$$

For the solutions corresponding to $\mu_{(1,2)}= \pm 2 K(1$ refers to positive sign and 2 refers to negative sign, respectively), $v_{x}$ can 
be written,

$$
\begin{aligned}
v_{x}^{(1,2)}= & \xi^{ \pm 2 K} \sum_{n=0}^{\infty} \frac{\left(a_{(1,2) 1}\right)_{n}\left(a_{(1,2) 2}\right)_{n}\left(-\xi^{2}\right)^{n}}{\left(b_{(1,2) 1}\right)_{n}\left(b_{(1,2) 2}\right)_{n}\left(b_{(1,2) 3}\right)_{n} n !} \\
& \times\left(\frac{\Omega^{2}}{\gamma}+(n \pm K)(n+1 \pm K)\right) .
\end{aligned}
$$

In both cases the $n$th term of the Pochammer symbols $\left(b_{(1,2) 2}\right)_{n}$ and $\left(b_{(1,2) 3}\right)_{n}$ are such that their product is

$$
\begin{aligned}
& \left(b_{(1,2) 2}+(n-1)\right)\left(b_{(1,2) 3}+(n-1)\right) \\
& \quad=\left(\frac{1}{2}-i q_{0} \pm K+n\right)\left(\frac{1}{2}+i q_{0} \pm K+n\right) \\
& \quad=\left(\frac{\Omega^{2}}{\gamma}+(n \pm K)(n+1 \pm K)\right) .
\end{aligned}
$$

It is then clear that the numerators and denominators of the $n$th terms cancel and the result is,

$$
\begin{aligned}
v_{x}^{(1,2)}= & \left(K^{2} \pm K+\frac{\Omega^{2}}{\gamma}\right) \xi^{ \pm 2 K} \\
& \times \sum_{n=0}^{\infty} \frac{\left(a_{(1,2) 1}\right)_{n}\left(a_{(1,2) 2}\right)_{n}\left(-\xi^{2}\right)^{n}}{\left(b_{(1,2) 1}\right)_{n}\left(b_{(1,2) 2}-1\right)_{n}\left(b_{(1,2) 3}-1\right)_{n} n !}
\end{aligned}
$$

Therefore, the type 1 and 2 solutions, for $v_{x}$, can be written as single hypergeometric functions. Considering now the solutions corresponding to $\mu_{(3,4)}=-1 \pm i 2 q_{0}$ (3 refers to positive sign and 4 refers to negative sign respectively), $v_{x}$ can be written,

$$
\begin{aligned}
v_{x}^{(3,4)}= & \xi^{-1 \pm 2 i q_{0}} \sum_{n=0}^{\infty} \frac{\left(a_{(3,4) 1}\right)_{n}\left(a_{(3,4) 2}\right)_{n}\left(-\xi^{2}\right)^{n}}{\left(b_{(3,4) 1}\right)_{n}\left(b_{(3,4) 2}\right)_{n}\left(b_{(3,4) 3}\right)_{n} n !} \\
& \times\left(\frac{\Omega^{2}}{\gamma}+\left(n-\frac{1}{2} \pm i q_{0}\right)\left(n+\frac{1}{2} \pm i q_{0}\right)\right) \\
= & \xi^{-1 \pm 2 i q_{0}} \sum_{n=0}^{\infty} \frac{\left(a_{(3,4) 1}\right)_{n}\left(a_{(3,4) 2}\right)_{n}\left(-\xi^{2}\right)^{n}}{\left(b_{(3,4) 1}\right)_{n}\left(b_{(3,4) 2}\right)_{n}\left(b_{(3,4) 3}\right)_{n} n !}\left(n \pm 2 i q_{0}\right) \\
= & \xi^{1 \pm 2 i q_{0}} \frac{\left(-a_{(3,4) 1} a_{(3,4) 2}\right)}{\left(b_{(3,4) 1} b_{(3,4) 2} b_{(3,4) 3}\right)} \\
& \times \sum_{n=0}^{\infty} \frac{\left(a_{(3,4) 1}+1\right)_{n}\left(a_{(3,4) 2}+1\right)_{n}\left(-\xi^{2}\right)^{n}(n+1)}{\left(b_{(3,4) 1}+1\right)_{n}\left(b_{(3,4) 2}+1\right)_{n}\left(b_{(3,4) 3}+1\right)_{n}(n+1) !} \\
& \times\left(n+1 \pm 2 i q_{0}\right) \\
= & \xi^{1 \pm 2 i q_{0}} \frac{\left(-a_{(3,4) 1} a_{(3,4) 2}\right)}{\left(b_{(3,4) 1} b_{(3,4) 2} b_{(3,4) 3}\right)} \\
& \times \sum_{n=0}^{\infty} \frac{\left(a_{(3,4) 1}+1\right)_{n}\left(a_{(3,4) 2}+1\right)_{n}\left(-\xi^{2}\right)^{n}}{\left(b_{(3,4) 1}+1\right)_{n}\left(b_{(3,4) 2}+1\right)_{n}\left(b_{(3,4) 3}+1\right)_{n} n !} \\
& \times\left(n+1 \pm 2 i q_{0}\right) .
\end{aligned}
$$

As the $n$th term of the Pochammer symbol $\left(b_{(3,4) 1}+1\right)_{n}$ is given as $\left(1 \pm 2 i q_{0}+n\right)$ and this is the same as the numerator, it is therefore possible to write,

$$
\begin{aligned}
v_{x}^{(3,4)}= & \xi^{1 \pm 2 i q_{0}} \frac{\left(-a_{(3,4) 1} a_{(3,4) 2}\right)}{\left(b_{(3,4) 2} b_{(3,4) 3}\right)} \\
& \times \sum_{n=0}^{\infty} \frac{\left(a_{(3,4) 1}+1\right)_{n}\left(a_{(3,4) 2}+1\right)_{n}\left(-\xi^{2}\right)^{n}}{\left(b_{(3,4) 1}+1\right)_{n}\left(b_{(3,4) 2}+1\right)_{n}\left(b_{(3,4) 3}+1\right)_{n} n !} .
\end{aligned}
$$

Again, the type 3 and 4 solutions for $v_{x}$ can be written as single hypergeometric functions multiplied by a power function. It is interesting to note that these solutions for the type 1,2,3, and 4 solutions are exactly the same as derived in Zhugzhda (1979). We can therefore write,

$$
\begin{aligned}
& v_{x}=C_{1} \xi^{2}{ }^{K} S^{(1)}{ }_{2} F_{3}\left[\begin{array}{c}
a_{11}, a_{12} ; \\
b_{11}, b_{12}-1, b_{13}-1
\end{array} \mid-\xi^{2}\right] \\
& +C_{2} \xi^{-2 K_{S}(2)}{ }_{2} F_{3}\left[\begin{array}{c}
a_{21}, a_{22} ; \\
b_{21}, b_{22}-1, b_{23}-1
\end{array} \mid-\xi^{2}\right] \\
& -C_{3} \xi^{1+2 i q_{0}} S^{(3)}{ }_{2} F_{3}\left[\begin{array}{c}
a_{31}+1, a_{32}+1 ; \\
b_{31}, b_{32}+1, b_{33}+1
\end{array} \mid-\xi^{2}\right] \\
& -C_{4} \xi^{1-2 i q_{0} S^{(4)}}{ }_{2} F_{3}\left[\begin{array}{c}
a_{41}+1, a_{42}+1 ; \\
b_{41}, b_{42}+1, b_{43}+1
\end{array} \mid-\xi^{2}\right] \\
& =\sum_{j=1}^{4} C_{j} v_{x}^{(j)}
\end{aligned}
$$

noting the factor

$$
\frac{\omega}{k_{x}\left(\omega^{2}-N^{2}\right)} \frac{1}{H^{2}}
$$

has been merged into the constants of integration and that,

$$
\begin{aligned}
& S^{(1)}=K^{2}+K+\frac{\Omega^{2}}{\gamma}, \quad S^{(2)}=K^{2}-K+\frac{\Omega^{2}}{\gamma}, \\
& S^{(3)}=\frac{a_{31} a_{32}}{b_{32} b_{33}}, \quad S^{(4)}=\frac{a_{41} a_{42}}{b_{42} b_{43}} .
\end{aligned}
$$

These solutions correspond exactly to those derived in Zhugzhda (1979) and Cally (2001). Having found the complete solution for $v_{x}$ it is prudent to find the solution for $v_{z}$ in the simplest possible terms. Transforming to the variable $\xi^{2}$ and dimensionless quantities, Equation (40) becomes,

$$
v_{z}=\frac{i \omega}{\omega^{2}-N^{2}} \frac{1}{H}\left(\xi^{2} \Theta^{\prime}+n^{2} \Theta\right) .
$$

It is then possible to write for each $\mu_{j}$,

$$
\begin{aligned}
v_{z}^{(j)} & =\xi^{\mu_{j}} \sum_{n=0}^{\infty} \frac{\left(a_{j}\right)_{n}\left(b_{j}\right)_{n}\left(-\xi^{2}\right)^{n}}{\left(c_{j}\right)_{n}\left(d_{j}\right)_{n}\left(e_{j}\right)_{n} n !}\left(\frac{\mu_{j}}{2}+n+\hat{N}^{2}\right) \\
& =\left(\frac{\mu_{j}}{2}+\hat{N}^{2}\right) \xi^{\mu_{j}} \sum_{n=0}^{\infty} \frac{\left(a_{j}\right)_{n}\left(b_{j}\right)_{n}\left(\frac{\mu_{j}}{2}+\hat{N}^{2}+1\right)_{n}\left(-\xi^{2}\right)^{n}}{\left(c_{j}\right)_{n}\left(d_{j}\right)_{n}\left(e_{j}\right)_{n}\left(\frac{\mu_{j}}{2}+\hat{N}^{2}\right)_{n} n !} .
\end{aligned}
$$

The solutions for $v_{z}$ in terms of hypergeometric functions is then,

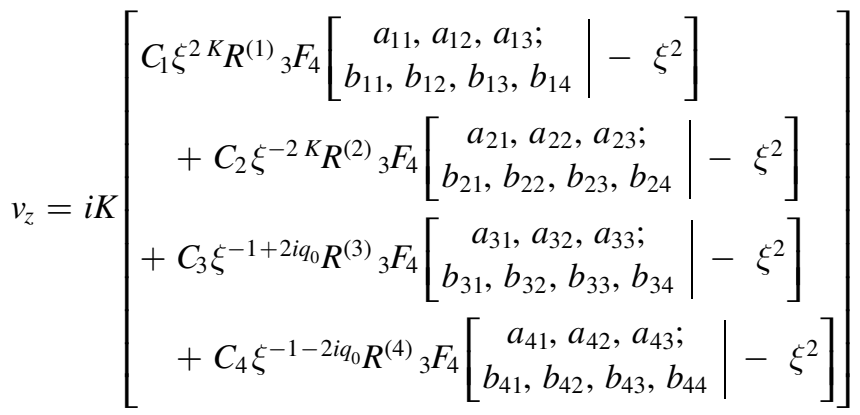


where,

$$
\begin{gathered}
R^{(1)}=\hat{N}^{2}+K, \quad R^{(2)}=\hat{N}^{2}-K, \\
R^{(3)}=\hat{N}^{2}-\frac{1}{2}+i q_{0}, \quad R^{(4)}=\hat{N}^{2}-\frac{1}{2}-i q_{0}, \\
a_{13}=R^{(1)}+1, \quad a_{23}=R^{(2)}+1, \\
a_{33}=R^{(3)}+1, \quad a_{23}=R^{(4)}+1, \\
b_{13}=R^{(1)}, \quad b_{23}=R^{(2)}, \quad b_{33}=R^{(3)}, \quad b_{43}=R^{(4)} .
\end{gathered}
$$

It must be noted that both Equations (45) and (47) are not valid when the difference of the quantities $2 K,-2 K, 1+2 i q_{0}$, and $1-2 i q_{0}$ is an integer number, as two of the solutions will no longer be linearly independent. If this was the case, logarithmic solutions would have to be derived. We therefore avoid points of this type in our analysis. We also note that these solutions converge absolutely for any given $\xi$. This can be verified simply by the ratio test.

\section{REFERENCES}

Abdelatif, T. E. 1990, SoPh, 129, 201

Banerjee, D., Hasan, S. S., \& Christensen-Dalsgaard, J. 1995, ApJ, 451, 825

Bray, R. J., \& Loughhead, R. E. 1974, The Solar Chromosphere (London: Chapman and Hall)

Cally, P. S. 2001, ApJ, 548, 473

Cowling, T. G. 1941, MNRAS, 101, 367

Fedun, V., Erdélyi, R., \& Shelyag, S. 2009, SoPh, 258, 219

Fedun, V., Shelyag, S., \& Erdélyi, R. 2011, ApJ, 727, 17

Ferraro, C. A., \& Plumpton, C. 1958, ApJ, 127, 459

Goedbloed, J. P. H., \& Poedts, S. 2004, Principles of Magnetohydrodynamics (Cambridge: Cambridge Univ. Press)

Hasan, S. S., \& Christensen-Dalsgaard, J. 1992, ApJ, 396, 311

Leroy, B., \& Schwartz, S. J. 1982, A\&A, 112, 84

Luke, Y. L. 1975, Mathematical Functions and Their Approximations (New York: Academic Press)

Miles, A. J., \& Roberts, B. 1992, SoPh, 141, 205

Moreno-Insertis, F., \& Spruit, H. C. 1989, ApJ, 342, 1158

Pintér, B. 1999, PhD thesis, Katholieke Universiteit Leuven Roberts, B. 2006, RSPTA, 364, 447

Scheuer, M. A., \& Thomas, J. H. 1981, SoPh, 71, 21

Schwartz, S. J., \& Leroy, B. 1982, A\&A, 112, 93

Yelles Chaouche, L., \& Abdelatif, T. E. 2005, SoPh, 229, 255

Zhugzhda, I. D., \& Dzhalilov, N. S. 1982, A\&A, 112, 16

Zhugzhda, Y. D. 1979, SvA, 23, 42 